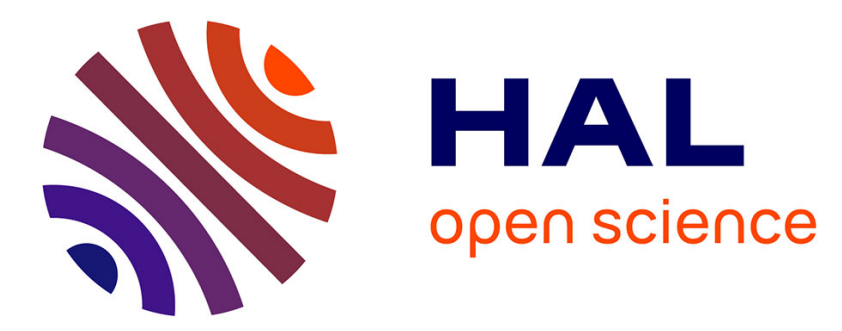

\title{
Enlargement of filtration in discrete time
}

Christophette Blanchet-Scalliet, Monique Jeanblanc, Ricardo Romo Roméro

\section{To cite this version:}

Christophette Blanchet-Scalliet, Monique Jeanblanc, Ricardo Romo Roméro. Enlargement of filtration in discrete time. Pauline Barrieu. Risk And Stochastics: Ragnar Norberg, Worl Scientific, pp.99-126, 2019, 9781786341945. hal-01253214

\section{HAL Id: hal-01253214 \\ https://hal.science/hal-01253214}

Submitted on 8 Jan 2016

HAL is a multi-disciplinary open access archive for the deposit and dissemination of scientific research documents, whether they are published or not. The documents may come from teaching and research institutions in France or abroad, or from public or private research centers.
L'archive ouverte pluridisciplinaire $\mathbf{H A L}$, est destinée au dépôt et à la diffusion de documents scientifiques de niveau recherche, publiés ou non, émanant des établissements d'enseignement et de recherche français ou étrangers, des laboratoires publics ou privés. 


\title{
Enlargement of filtration in discrete time
}

\author{
C. Blanchet-Scalliet $\stackrel{*}{,}$ M. Jeanblanc $\stackrel{\dagger}{\dagger}$ R. Romo Romero ${ }^{\ddagger}$
}

January 8, 2016

\begin{abstract}
We present some results on enlargement of filtration in discrete time. Many results known in continuous time extend immediately in a discrete time setting. Here, we provide direct proofs which are much more simpler. We study also arbitrages conditions in a financial setting and we present some specific cases, as immersion and pseudo-stopping times for which we obtain new results.
\end{abstract}

\section{Introduction}

In this paper, we present classical results on enlargement of filtration, in a discrete time framework. In such a setting, any $\mathbb{F}$-martingale is a semimartingale for any filtration $\mathbb{G}$ larger than $\mathbb{F}$, and one can think that there are not so many things to do. From our point of view, one interest of our paper is that the proofs of the semimartingale decomposition formula are simple, and give a pedagogical support to understand the general formulae obtained in the literature in continuous time. It can be noted that many results are established in continuous time under the hypothesis that all $\mathbb{F}$-martingales are continuous or, in the progressive enlargement case, that the random time avoids the $\mathbb{F}$-stopping times and the extension to the general case is difficult. In discrete time, one can not make any of such assumptions, since martingales are discontinuous and random times valued in the set of integers do not avoid $\mathbb{F}$-stopping times.

In the first section, we recall some well know facts. Section 2 is devoted to the case of initial enlargement. Section 3 presents the case of progressive enlargement with a random time $\tau$. We give a "model-free" definition of arbitrages in the context of enlargement of filtration, we study some examples in initial enlargement and give, in a progressive enlargement setting, necessary and sufficient conditions to avoid arbitrages before $\tau$. We present the particular case of honest times (which are the standard example in continuous time) and we give conditions to obtain immersion property. We also give also various characterizations of pseudo-stopping times.

\section{Some well known Results and Definitions}

In this paper, we are working in a discrete time setting: $X=\left(X_{n}, n \geq 0\right)$ is a process on a probability space $(\Omega, \mathbb{P})$, and $\mathbb{H}=\left(\mathcal{H}_{n}, n \geq 0\right)$ is a filtration. We note $\Delta X_{n}:=X_{n}-X_{n-1}, n \geq 1$

${ }^{*}$ C. B-S. Université de Lyon - CNRS, UMR 5208, Institut Camille Jordan - Ecole Centrale de Lyon, 36 avenue Guy de Collongue, 69134 Ecully Cedex, FRANCE.

${ }^{\dagger}$ M.J., R.R.: LaMME, UMR CNRS 8071, Université d'Evry-Val-D’Essonne, 23 boulevard de France, 91037 Evry

¥The research of M. Jeanblanc and R. Romo Romero is supported by the Chaire Marchés en Mutation (Fédération Bancaire Française). M.J. thanks K. Kardaras for fruitful discussion on arbitrages opportunities. 
the increment of $X$ at time $n$ and we set $\Delta X_{0}=X_{0}$. A process $X$ is $\mathbb{H}$-predictable if, for any $n \geq 1$, the random variable $X_{n}$ is $\mathcal{H}_{n-1}$-measurable and $X_{0}$ is a constant. A process $X$ is square integrable if $\mathbb{E}\left(X_{n}^{2}\right)<\infty$ for all $n \geq 0$. A random variable $X$ is positive if $X>0$ a.s. and a process $A$ is increasing (resp. decreasing) if $A_{n} \geq A_{n-1}$ (resp. $A_{n} \leq A_{n-1}$ ) a.s. , for all $n \geq 1$.

\section{$1.1 \quad \mathbb{H}$-martingales}

We give some obvious results on the form of $\mathbb{H}$-martingales.

The set of processes of the form $\left(\psi_{0}+\sum_{k=1}^{n} \psi_{k}-\mathbb{E}\left(\psi_{k} \mid \mathcal{H}_{k-1}\right), n \geq 0\right)$ where $\psi$ is an $\mathbb{H}-$ adapted integrable process is equal to the set of all $\mathbb{H}$-martingales (here, $\sum_{k=1}^{0} \cdot=0$ )

The set of processes of the form $\left(\psi_{0} \prod_{k=1}^{n} \frac{\psi_{k}}{\mathbb{E}\left(\psi_{k} \mid \mathcal{H}_{k-1}\right)}, n \geq 0\right)$ where $\psi$ is a positive integrable $\mathbb{H}$-adapted process is the set of all positive $\mathbb{H}$-martingales (here, $\prod_{k=1}^{0} \cdot=1$ ).

\subsection{Doob's Decomposition and Applications}

\subsubsection{Doob's decomposition}

Lemma 1.1 Any integrable $\mathbb{H}$-adapted process $X$ is a special $\mathbb{H}$-semimartingale ${ }^{1}$ with (unique) decomposition $X=M^{X, \mathbb{H}}+V^{X, \mathbb{H}}$ where $M^{X, \mathbb{H}}$ is an $\mathbb{H}$-martingale and $V^{X, \mathbb{H}}$ is an $\mathbb{H}$-predictable process with $V_{0}^{X, \mathbb{H}}=0$. Furthermore,

$$
\Delta V_{n}^{X, \mathbb{H}}=\mathbb{E}\left(\Delta X_{n} \mid \mathcal{H}_{n-1}\right), \forall n \geq 1 .
$$

Proof. In the proof, $V^{\mathbb{H}}:=V^{X, \mathbb{H}}$ and $M^{\mathbb{H}}:=M^{X, \mathbb{H}}$. Setting $V_{0}^{\mathbb{H}}=0$ and, for $n \geq 1$

$$
V_{n}^{\mathbb{H}}-V_{n-1}^{\mathbb{H}}=\mathbb{E}\left(X_{n}-X_{n-1} \mid \mathcal{H}_{n-1}\right),
$$

we construct an $\mathbb{H}$-predictable process. This leads to $\Delta M_{n}^{\mathbb{H}}=\Delta X_{n}-\Delta V_{n}^{\mathbb{H}}=X_{n}-\mathbb{E}\left(X_{n} \mid \mathcal{H}_{n-1}\right), \forall n \geq$ 1. Setting $M_{0}^{\mathbb{H}}=X_{0}$, the process $M^{\mathbb{H}}$ is an $\mathbb{H}$-martingale.

In what follows, we shall also denote $V^{X}$ (resp. $\left.V^{\mathbb{H}}\right)$ the $\mathbb{H}$-predictable part of $X$ if there are no ambiguity on the choice of the filtration (resp. on the choice of the process).

As an immediate corollary, we obtain the Doob decomposition of supermartingales:

Corollary 1.2 If $X$ is an $\mathbb{H}$-adapted supermartingale, it admits a unique decomposition

$$
X=M^{X}-A^{X}
$$

where $M^{X}$ is an $\mathbb{H}$-martingale and $A^{X}$ is an increasing $\mathbb{H}$-predictable process with $A^{X}=0$.

\subsubsection{Predictable brackets}

Proposition 1.3 If $X$ and $Y$ are square integrable $\mathbb{H}$-martingales, there exists a unique $\mathbb{H}$ predictable process $V^{X, Y}=:\langle X, Y\rangle$ such that $V_{0}^{X, Y}=0$ and $X Y-V^{X, Y}$ is an $\mathbb{H}$-martingale. Furthermore

$$
\Delta V_{n}^{X, Y}=\mathbb{E}\left(Y_{n} \Delta X_{n} \mid \mathcal{H}_{n-1}\right)=\mathbb{E}\left(\Delta Y_{n} \Delta X_{n} \mid \mathcal{H}_{n-1}\right), n \geq 1 .
$$

Proof. Indeed, from Lemma 1.1, and using the martingale property of $X$ and $Y$, we have, for $n \geq 1$ :

$$
\begin{aligned}
\Delta V_{n}^{X, Y}=V_{n}^{X, Y}-V_{n-1}^{X, Y} & =\mathbb{E}\left(X_{n} Y_{n}-X_{n-1} Y_{n-1} \mid \mathcal{H}_{n-1}\right) \\
& =\mathbb{E}\left(Y_{n} \Delta X_{n} \mid \mathcal{H}_{n-1}\right)+\mathbb{E}\left(X_{n-1} \Delta Y_{n} \mid \mathcal{H}_{n-1}\right)=\mathbb{E}\left(Y_{n} \Delta X_{n} \mid \mathcal{H}_{n-1}\right) \\
& =\mathbb{E}\left(\Delta Y_{n} \Delta X_{n} \mid \mathcal{H}_{n-1}\right) .
\end{aligned}
$$

\footnotetext{
${ }^{1}$ A special semimartingale is an adapted process $X$ such that $X=M+V$ where $M$ is a martingale and $V$ a predictable process.
} 
The predictable bracket of two semimartingales $X, Y$ is defined in continuous time as the dual predictable projection of the covariation process, that is $\langle X, Y\rangle:=[X, Y]^{p}$. For discrete time semimartingales, we adopt the same definition. The covariation process is

$$
[X, Y]_{0}=0,[X, Y]_{n}:=\sum_{k=1}^{n} \Delta X_{k} \Delta Y_{k}, n \geq 1,
$$

and $[X, Y]^{p}$ is the unique predictable (bounded variation) process null at time 0 , such that $[X, Y]-[X, Y]^{p}$ is a martingale, i.e., $[X, Y]^{p}$ is the predictable part of the semimartingale $[X, Y]$.

Lemma 1.4 Let $X, Y$ be two $\mathbb{H}$-adapted processes (hence, semimartingales). Then

$$
\langle X, Y\rangle_{0}=0, \Delta\langle X, Y\rangle_{n}=\mathbb{E}\left(\Delta X_{n} \Delta Y_{n} \mid \mathcal{H}_{n-1}\right), n \geq 1
$$

Proof. From Doob's decomposition (Lemma 1.1), for $n \geq 1$,

$$
\left(\Delta[X, Y]^{p}\right)_{n}=\mathbb{E}\left([X, Y]_{n}-[X, Y]_{n-1} \mid \mathcal{H}_{n-1}\right)=\mathbb{E}\left(\Delta X_{n} \Delta Y_{n} \mid \mathcal{H}_{n-1}\right) .
$$

As usual, two martingales $X$ and $Y$ are said to be orthogonal if the product is a martingale, i.e., if $\mathbb{E}\left(\Delta Y_{n} \Delta X_{n} \mid \mathcal{H}_{n-1}\right)=0, \forall n \geq 1$.

\subsubsection{Stochastic integral of optional processes and martingale property}

Lemma 1.5 If $Y$ is an $\mathbb{H}$-adapted square integrable process and $X$ a square integrable $\mathbb{H}-$ martingale, the process $Y \cdot X$ defined as $(Y \cdot X)_{n}:=Y_{0}+\sum_{k=1}^{n} Y_{k} \Delta X_{k}, n \geq 0$ is an $\mathbb{H}$-martingale if and only if the $\mathbb{H}$-martingale part of $Y$ is orthogonal to $X$. In particular, if $Y$ is $\mathbb{H}$-predictable, $Y \cdot X$ is an $\mathbb{H}$-martingale.

Proof. Let $Y=M^{Y}+V^{Y}$. Since

$$
\mathbb{E}\left(Y_{n} \Delta X_{n} \mid \mathcal{H}_{n-1}\right)=\mathbb{E}\left(M_{n}^{Y} \Delta X_{n} \mid \mathcal{H}_{n-1}\right)+V_{n}^{Y} \mathbb{E}\left(\Delta X_{n} \mid \mathcal{H}_{n-1}\right)=\mathbb{E}\left(\Delta M_{n}^{Y} \Delta X_{n} \mid \mathcal{H}_{n-1}\right)
$$

the result is obvious.

\subsection{Multiplicative decomposition}

Theorem 1.6 Let $X$ be an $\mathbb{H}$-adapted (integrable) positive process, then $X$ can be represented in a unique form as

$$
X=K^{X} N^{X}
$$

where $K^{X}$ is an $\mathbb{H}$-predictable process with $K_{0}^{X}=1$ and $N^{X}$ is an $\mathbb{H}$-martingale. More precisely,

$$
\begin{array}{cl}
N_{0}^{X}=X_{0}, & N_{n}^{X}=X_{0} \prod_{k=1}^{n} \frac{X_{k}}{\mathbb{E}\left(X_{k} \mid \mathcal{H}_{k-1}\right)}, \quad \forall n \geq 1, \\
K_{0}^{X}=1, & K_{n}^{X}=\prod_{k=1}^{n} \frac{\mathbb{E}\left(X_{k} \mid \mathcal{H}_{k-1}\right)}{X_{k-1}}, \quad \forall n \geq 1 .
\end{array}
$$

Proof. For each $n \geq 1$ fixed, the positive random variable $N_{n}^{X}$, is integrable since by recurrence $\mathbb{E}\left[N_{n}^{X}\right]=\mathbb{E}\left[N_{n-1}^{X} \mathbb{E}\left(\frac{X_{n}}{\mathbb{E}\left(X_{n} \mid \mathcal{H}_{n-1}\right)} \mid \mathcal{H}_{n-1}\right)\right]=\mathbb{E}\left(N_{n-1}^{X}\right)=X_{0}$, and from Subsection $1.1, N^{X}$ is a martingale.

In the other hand, the process $K^{X}$, defined by

is an $\mathbb{H}$-predictable process.

$$
K_{n}^{X}=\frac{X_{n}}{X_{0} \prod_{k=1}^{n} \frac{X_{k}}{\mathbb{E}\left(X_{k} \mid \mathcal{H}_{k-1}\right)}}=\prod_{k=1}^{n} \frac{\mathbb{E}\left(X_{k} \mid \mathcal{H}_{k-1}\right)}{X_{k-1}}
$$

Remark 1.7 In terms of Doob's decomposition $X=M^{X}+V^{X}$, one has

$$
K_{n}^{X}=\frac{M_{n-1}^{X}+V_{n}^{X}}{X_{n-1}} K_{n-1}^{X}=X_{0} \prod_{k=1}^{n} \frac{M_{k-1}^{X}+V_{k}^{X}}{X_{k-1}}, N_{n}^{X}=X_{n} / K_{n}^{X}, n \geq 1 .
$$

Corollary 1.8 Any positive $\mathbb{H}$-supermartingale $Y$ admits a unique multiplicative decomposition $Y=N D$ where $N$ is an $\mathbb{H}$-martingale and $D$ an $\mathbb{H}$-predictable decreasing process with $D=1$.

Proof. The process $D=K^{X}$ is indeed decreasing. 


\subsection{Exponential process}

Given an integrable process $X$, such that $X_{0}=0$, we define the exponential of $X$ denoted by $\mathcal{E}(X)$ as the solution of the following equation in differences:

$$
\begin{cases}\Delta \mathcal{E}(X)_{n} & =\mathcal{E}(X)_{n-1} \Delta X_{n}, \quad \forall n \geq 1 \\ \mathcal{E}(X)_{0} & =1\end{cases}
$$

Proposition 1.9 The solution of (1), is given by

$$
\mathcal{E}(X)_{n}:=\Pi_{k=1}^{n}\left(\Delta X_{k}+1\right), \quad \forall n \geq 1 .
$$

If $\Delta X_{n}>-1$, for all $n \geq 0$, then $\mathcal{E}(X)$ is positive.

Lemma 1.10 Let $\psi$ and $\gamma$ be predictable and $M$ and $N$ be two martingales. Then

$$
\mathcal{E}(\psi \cdot M) \mathcal{E}(\gamma \cdot N)=\mathcal{E}(\psi \cdot M+\gamma \cdot N+\psi \gamma \cdot[M, N]) .
$$

Proof. This result is known as Yor's equality. By definition, the two sides are equal to 1 at time 0 . For $n \geq 1$, the left-hand side $K_{n}:=\mathcal{E}(\psi \cdot M)_{n} \mathcal{E}(\gamma \cdot N)_{n}$ satisfies $K_{n}=K_{n-1}(1+$ $\left.\psi_{n} \Delta M_{n}\right)\left(1+\gamma_{n} \Delta N_{n}\right)$. The right-hand side $J_{n}:=\mathcal{E}(\psi \cdot M+\gamma \cdot N+\psi \gamma \cdot[M, N])_{n}$ satisfies $J_{n}=J_{n-1}\left(1+\psi_{n} \Delta M_{n}+\gamma_{n} \Delta N_{n}+\psi_{n} \gamma_{n} \Delta M_{n} \Delta N_{n}\right)$. Assuming by recurrence that $K_{n-1}=J_{n-1}$, the result follows.

\subsection{Girsanov's transformation}

Theorem 1.11 Let $X$ be a $(\mathbb{P}, \mathbb{H})$-martingale, $\mathbb{Q}$ a probability measure such that $\mathbb{Q} \sim \mathbb{P}$ on $\mathcal{H}_{n}$ for all $n \geq 0$, and $L_{n}:=\left.\frac{d \mathbb{Q}}{d \mathbb{P}}\right|_{\mathcal{H}_{n}}$. If $X_{n} \in L^{1}(\mathbb{Q})$ for all $n \geq 0$, then the process $\tilde{X}$ defined as

$$
\widetilde{X}_{n}=X_{n}-\sum_{k=1}^{n} \frac{1}{L_{k-1}} \Delta\langle X, L\rangle_{k}, \forall n \geq 0
$$

is a $\mathbb{Q}$-martingale.

Proof. The result follows from the fact that, from Doob's decomposition, the process $X^{\mathbb{Q}}$ defined, for $n \geq 0$ as

$$
X_{n}^{\mathbb{Q}}=X_{n}-\sum_{k=1}^{n} \mathbb{E}_{\mathbb{Q}}\left[\Delta X_{k} \mid \mathcal{H}_{k-1}\right]=X_{n}-\sum_{k=1}^{n} \frac{1}{L_{k-1}} \mathbb{E}_{\mathbb{P}}\left[L_{k} \Delta X_{k} \mid \mathcal{H}_{k-1}\right]
$$

is a $\mathbb{Q}$-martingale.

\subsection{Enlargement of filtration}

In continuous time, a difficult problem is to give conditions such that an $\mathbb{F}$-martingale is a $\mathbb{G}$-semimartingale for two filtrations satisfying $\mathbb{F} \subset \mathbb{G}$, and, if it is the case, to give the $\mathbb{G}$ semimartingale decomposition of an $\mathbb{F}$-martingale. In discrete time, the following proposition is an easy consequence of Doob's decomposition and states that if $\mathbb{F} \subset \mathbb{G}$, then any $\mathbb{F}$-martingale is a $\mathbb{G}$-semimartingale and gives explicitly the decomposition of this semimartingale.

Proposition 1.12 In a discrete time setting, any integrable process is a special semimartingale in any filtration with respect to which it is adapted: if $\mathbb{F} \subset \mathbb{G}$, and if $X$ is an $\mathbb{F}$-martingale, it is $a \mathbb{G}$ special semimartingale with decomposition

$$
X=M^{\mathbb{G}}+V^{\mathbb{G}}
$$

where $M^{\mathbb{G}}$ is a $\mathbb{G}$-martingale and $V^{\mathbb{G}}$ is $\mathbb{G}$-predictable, $V_{0}^{\mathbb{G}}=0$, and

$$
\Delta V_{n}^{\mathbb{G}}=\mathbb{E}\left(\Delta X_{n} \mid \mathcal{G}_{n-1}\right), n \geq 1 .
$$

Our goal is to compute more explicitly the semimartingale decomposition in some specific cases, and to show, with elementary computations, that we recover the classical general formulae established in the literature in continuous time. 
Comment 1.13 Note that results in continuous time can be directly applied to discrete time: if $\mathbb{F}$ is a discrete time filtration and $X$ a discrete time process, one can study the continuous on right jumping filtration $\widetilde{\mathbb{F}}$ defined in continuous time for $n \leq t<n+1$ as $\widetilde{\mathcal{F}}_{t}=\mathcal{F}_{n}$, and the càdlàg process $\widetilde{X}_{t}=\sum_{n} X_{n} \mathbb{1}_{\{n \leq t<n+1\}}$. One interest of our computations relies on the fact that we do not need hypotheses done in continuous time and that our proofs are simple.

Another goal of this paper is to study how enlarging the filtration may introduce arbitrages. To do this, we first recall some definition of no arbitrage for a given filtration

Definition 1.14 Let $X$ be an $\mathbb{H}$-semimartingale, where $\mathbb{H}$ is a given filtration. We say that the model $(X, \mathbb{H})$ has no arbitrages if there exists a positive $\mathbb{H}$-martingale $L$, with $L_{0}=1$, such that $X L$ is an $\mathbb{H}$-martingale.

We start with a general result, valid for any filtration $\mathbb{H}$ :

Lemma 1.15 Let $Y$ be an integrable $\mathbb{H}$-semimartingale. If there exists a positive $\mathbb{H}$-adapted process $\psi$ such that

$$
\mathbb{E}\left(Y_{n} \psi_{n} \mid \mathcal{H}_{n-1}\right)=Y_{n-1} \mathbb{E}\left(\psi_{n} \mid \mathcal{H}_{n-1}\right), \forall n \geq 1,
$$

there exists a positive $\mathbb{H}$-martingale $L$ such that $L Y$ is an $\mathbb{H}$-martingale.

Proof. Let $Y$ be a $(\mathbb{P}, \mathbb{H})$-semimartingale with decomposition $Y=M^{Y}+V^{Y}$, with $\Delta V_{n}^{Y}=$ $\mathbb{E}\left(\Delta Y_{n} \mid \mathcal{H}_{n-1}\right)$ and where $M^{Y}$ is a $(\mathbb{P}, \mathbb{H})$-martingale. Define, for a given $\psi$, the $(\mathbb{P}, \mathbb{H})$-martingale $L$

$$
L_{0}=1, L_{n}=\prod_{k=1}^{n} \frac{\psi_{k}}{\mathbb{E}\left(\psi_{k} \mid \mathcal{H}_{k-1}\right)}=L_{n-1} \frac{\psi_{n}}{\mathbb{E}\left(\psi_{n} \mid \mathcal{H}_{n-1}\right)}, n \geq 1,
$$

then, setting $d \mathbb{Q}=L d \mathbb{P}$, the process $M^{Y}$ decomposes as $M^{Y}=m^{M}+V^{M}$ where $m^{M}$ is a $(\mathbb{Q}, \mathbb{H})$-martingale and

$$
\begin{aligned}
\Delta V_{n}^{M} & =\mathbb{E}_{\mathbb{Q}}\left(\Delta M_{n}^{Y} \mid \mathcal{H}_{n-1}\right)=\frac{1}{L_{n-1}} \mathbb{E}_{\mathbb{P}}\left(L_{n} \Delta M_{n}^{Y} \mid \mathcal{H}_{n-1}\right) \\
& =\frac{1}{L_{n-1}}\left(\mathbb{E}_{\mathbb{P}}\left(L_{n} M_{n}^{Y} \mid \mathcal{H}_{n-1}\right)-L_{n-1} M_{n-1}^{Y}\right)=\frac{1}{\mathbb{E}_{\mathbb{P}}\left(\psi_{n} \mid \mathcal{H}_{n-1}\right)} \mathbb{E}_{\mathbb{P}}\left(\psi_{n} \Delta M_{n}^{Y} \mid \mathcal{H}_{n-1}\right) .
\end{aligned}
$$

The process $Y$ is a $(\mathbb{Q}, \mathbb{H})$-martingale if $V^{M}+V^{Y}=0$ or equivalently $\Delta V^{Y}+\Delta V^{M}=0$, that is

$$
\mathbb{E}\left(\psi_{n} \Delta M_{n}^{Y} \mid \mathcal{H}_{n-1}\right)+\mathbb{E}\left(\psi_{n} \mid \mathcal{H}_{n-1}\right) \mathbb{E}\left(\Delta Y_{n} \mid \mathcal{H}_{n-1}\right)=0 .
$$

We develop and use that $\Delta M_{n}^{Y}=Y_{n}-\mathbb{E}\left(Y_{n} \mid \mathcal{H}_{n-1}\right)$ and obtain, after simplification

$$
\mathbb{E}\left(\psi_{n} Y_{n} \mid \mathcal{H}_{n-1}\right)=\mathbb{E}\left(\psi_{n} \mid \mathcal{H}_{n-1}\right) Y_{n-1}
$$

In the setting of enlargement of filtration, we introduce the following "model free" definition

Definition 1.16 Let $\mathbb{F} \subset \mathbb{G}$, we say that the model $(\mathbb{F}, \mathbb{G})$ is arbitrage free if there exists a positive $\mathbb{G}$-martingale $L$ with $L_{0}=1$ (called a deflator) such that, for any $\mathbb{F}$-martingale $X$, the process $X L$ is a $\mathbb{G}$-martingale.

Our definition is "model free" in the sense that we do not specify the price process in the filtration $\mathbb{F}$. The study of conditions so that, for a given martingale $X$, there exists a deflator, can be found in Choulli and Deng [7]. In the enlargement of filtration setting, we assume that there are no arbitrages in $\mathbb{F}$, and we work under a risk neutral probability measure in the filtration $\mathbb{F}$. Working under the historical probability does not create problems: it suffices to change the probability at the beginning.

\section{Initial Enlargement}

The filtration $\mathbb{G}=\left(\mathcal{G}_{n}, n \geq 0\right)$ is an initial enlargement of $\mathbb{F}$ with a random variable $\xi$ taken values in $\mathbb{R}$ if $\mathcal{G}_{n}:=\mathcal{F}_{n} \vee \sigma(\xi), n \geq 0$. 


\subsection{Bridge}

We study the following particular example. Let $\left(Y_{i}, i \geq 1\right)$ a sequence of i.i.d. random variables with zero mean and the process $X$ of the form $X_{0}:=0, X_{n}:=\sum_{i=1}^{n} Y_{i}, n \geq 1$. For $N$ fixed, we put $\xi:=X_{N}$ and we denote by $\mathbb{F}$ the natural filtration of $X$, and we note that $X$ is an $\mathbb{F}$-martingale.

We need to compute $\Delta V_{n}=\mathbb{E}\left(\Delta X_{n} \mid \mathcal{F}_{n-1} \vee \sigma\left(X_{N}\right)\right)$. Using the fact that $\left(Y_{i}, i \geq 1\right)$ are i.i.d, we have, for $n \leq j \leq N$

$$
\left(Y_{j}, X_{1}, \cdots, X_{n-1}, X_{N}\right) \stackrel{\text { law }}{=}\left(Y_{n}, X_{1}, \cdots, X_{n-1}, X_{N}\right),
$$

hence

$$
\begin{aligned}
\mathbb{E}\left(Y_{n} \mid \mathcal{F}_{n-1} \vee \sigma\left(X_{N}\right)\right) & =\mathbb{E}\left(Y_{j} \mid \mathcal{F}_{n-1} \vee \sigma\left(X_{N}\right)\right) \\
& =\frac{1}{N-(n-1)} \mathbb{E}\left(Y_{n}+\cdots+Y_{j}+\cdots+Y_{N} \mid \mathcal{F}_{n-1} \vee \sigma\left(X_{N}\right)\right) \\
& =\frac{1}{N-(n-1)} \mathbb{E}\left(X_{N}-X_{n-1} \mid \mathcal{F}_{n-1} \vee \sigma\left(X_{N}\right)\right)=\frac{X_{N}-X_{n-1}}{N-(n-1)}
\end{aligned}
$$

Therefore, the process $X^{\mathbb{G}}$ defined as

$$
X_{n}^{\mathbb{G}}=X_{n}-\sum_{k=1}^{n} \frac{X_{N}-X_{k-1}}{N-(k-1)}, n \geq 0
$$

is a $\mathbb{G}$-martingale.

Comment 2.1 This formula is similar to the one obtained for Lévy bridges: if $X$ is an integrable Lévy process in continuous time (e.g. a Brownian motion) with natural filtration $\mathbb{F}^{X}$, setting $\mathbb{G}=\mathbb{F}^{X} \vee \sigma\left(X_{T}\right)$ leads to

$$
X_{t}^{\mathbb{G}}=X_{t}-\int_{0}^{t} \frac{X_{T}-X_{s}}{T-s} d s, 0 \leq t \leq T
$$

where $X^{\mathbb{G}}$ is a $\mathbb{G}$-martingale.

\subsection{Initial enlargement with $\xi$, a $\mathbb{Z}$-valued random variable}

Let $X$ be an $\mathbb{F}$-martingale, $\xi$ be a r.v. taking values in $\mathbb{Z}$ and, for any $j \in \mathbb{Z}$, let $p(j)$ be the $\mathbb{F}$-martingale defined as $p_{n}(j)=\mathbb{P}\left(\xi=j \mid \mathcal{F}_{n}\right)$.

Then, the Doob decomposition of $X$ in $\mathbb{G}$ is $X=M^{\mathbb{G}}+V^{\mathbb{G}}$ where $M^{\mathbb{G}}$ is a $\mathbb{G}$-martingale and, for $n \geq 1, \Delta V_{n}^{\mathbb{G}}=\mathbb{E}\left(\Delta X_{n} \mid \mathcal{F}_{n-1} \vee \sigma(\xi)\right)$ so that

$$
\begin{aligned}
\left(\Delta V_{n}^{\mathbb{G}}\right) \mathbb{1}_{\{\xi=j\}} & =\mathbb{1}_{\{\xi=j\}} \frac{\mathbb{E}\left(\mathbb{1}_{\{\xi=j\}} \Delta X_{n} \mid \mathcal{F}_{n-1}\right)}{\mathbb{P}\left(\xi=j \mid \mathcal{F}_{n-1}\right)} \\
& =\mathbb{1}_{\{\xi=j\}} \frac{\mathbb{E}\left(p_{n}(j) \Delta X_{n} \mid \mathcal{F}_{n-1}\right)}{p_{n-1}(j)}=\mathbb{1}_{\{\xi=j\}} \frac{\Delta\langle X, p(j)\rangle_{n}}{p_{n-1}(j)},
\end{aligned}
$$

where we have used the tower property in the second equality. On the set $\{\xi=j\}$, one has $p_{n}(j) \neq 0, \forall n \geq 0$. Indeed,

$$
\mathbb{E}\left(\mathbb{1}_{\left\{p_{n}(j)=0\right\}} \mathbb{1}_{\{\xi=j\}}\right)=\mathbb{E}\left(\mathbb{1}_{\left\{p_{n}(j)=0\right\}} \mathbb{E}\left(\mathbb{1}_{\{\xi=j\}} \mid \mathcal{F}_{n}^{X}\right)\right)=\mathbb{E}\left(\mathbb{1}_{\left\{p_{n}(j)=0\right\}} p_{n}(j)\right)=0 .
$$

Therefore, the process $X^{\mathbb{G}}$ defined as

$$
X_{n}^{\mathbb{G}}=X_{n}-\sum_{k=1}^{n} \frac{\left.\Delta\langle X, p(j)\rangle_{k}\right|_{\xi=j}}{p_{k-1}(\xi)}
$$

is a $\mathbb{G}$-martingale.

Comment 2.2 In continuous time, under Jacod's hypothesis $\mathbb{P}\left(\tau \in d u \mid \mathcal{F}_{t}\right)=p_{t}(u) \mathbb{P}(\tau \in d u)$, the process $X^{\mathbb{G}}$ is a $\mathbb{G}$-martingale where

$$
X_{t}^{\mathbb{G}}=X_{t}-\int_{0}^{t} \frac{\left.d\langle X, p(u)\rangle_{s}\right|_{\xi=u}}{p_{s-}(\xi)}, \quad \forall t \geq 0 .
$$




\subsection{Arbitrages}

Lemma 2.3 If $\xi \in \mathcal{F}_{N}$ for some $N$ and $\xi \notin \mathcal{F}_{0}$, the model $(\mathbb{F}, \mathbb{G})$ is not arbitrage free.

Proof. Let $X_{n}=\mathbb{E}\left(\xi \mid \mathcal{F}_{n}\right)$. If a $\mathbb{G}$-deflator $L$ exists, the process $X L$ would be a $\mathbb{G}$-martingale, and $X_{n} L_{n}=\mathbb{E}\left(X_{N} L_{N} \mid \mathcal{G}_{n}\right)$. Using the fact that $X_{N}=\xi \in \mathcal{G}_{n}, 0 \leq n \leq N$, we obtain $\mathbb{E}\left(X_{N} L_{N} \mid \mathcal{G}_{n}\right)=X_{N} L_{n}$, in particular $X_{N} L_{0}=X_{0} L_{0}$ which is not possible since $X_{N}=\xi$ is not in $\mathcal{F}_{0}$.

\section{Progressive Enlargement}

We assume that $\tau$ is a random variable valued in $\mathbb{N} \cup\{+\infty\}$, and introduce the filtration $\mathbb{G}$ where, for $n \geq 0$, we set $\mathcal{G}_{n}=\mathcal{F}_{n} \vee \sigma(\tau \wedge n)$. In particular $\{\tau=0\} \in \mathcal{G}_{0}$, so that, in general $\mathcal{G}_{0}$ is not trivial.

In continuous time, many results are obtained under the hypothesis that $\tau$ avoids $\mathbb{F}$-stopping times, or that all $\mathbb{F}$-martingales are continuous, which is not the case here. We present here some basic results, and we refer to Romo Romero [9] for more information.

\subsection{General results}

We now assume that $\mathcal{F}_{0}$ is trivial. If $Y$ is a $\mathbb{G}$-adapted process, then there exists an $\mathbb{F}$-adapted process $y$ such that

$$
Y_{n} \mathbb{1}_{\{n<\tau\}}=y_{n} \mathbb{1}_{\{n<\tau\}}, \forall n \geq 0 .
$$

If $Y$ is a $\mathbb{G}$-predictable process, there exists an $\mathbb{F}$-predictable process $y$ such that

$$
Y_{n} \mathbb{1}_{\{n \leq \tau\}}=y_{n} \mathbb{1}_{\{n \leq \tau\}}, \forall n \geq 0 .
$$

We introduce the supermartingale

$$
Z_{n}=\mathbb{P}\left(\tau>n \mid \mathcal{F}_{n}\right), \forall n \geq 0
$$

and its Doob's decomposition $Z=M-A$ with

$$
A_{0}=0, \Delta A_{n}=-\mathbb{E}\left(\Delta Z_{n} \mid \mathcal{F}_{n-1}\right)=\mathbb{P}\left(\tau=n \mid \mathcal{F}_{n-1}\right), \forall n \geq 1 .
$$

In particular, since $\mathcal{F}_{0}$ is trivial, $Z_{0}=M_{0}=\mathbb{P}(\tau>0)$.

We also introduce the supermartingale

$$
\widetilde{Z}_{n}=\mathbb{P}\left(\tau \geq n \mid \mathcal{F}_{n}\right), \forall n \geq 0
$$

and its Doob's decomposition $\widetilde{Z}=\widetilde{M}-\widetilde{A}$ where $\widetilde{M}$ is an $\mathbb{F}$-martingale and $\widetilde{A}$ the $\mathbb{F}$-predictable increasing process satisfying $\widetilde{A}_{0}=0, \Delta \widetilde{A}_{n}=\mathbb{P}\left(\tau=n-1 \mid \mathcal{F}_{n-1}\right), \forall n \geq 1$.

We shall often use the trivial equalities

$$
\widetilde{Z}_{n}=\mathbb{P}\left(\tau>n-1 \mid \mathcal{F}_{n}\right)=Z_{n}+\mathbb{P}\left(\tau=n \mid \mathcal{F}_{n}\right), Z_{n}=\mathbb{P}\left(\tau \geq n+1 \mid \mathcal{F}_{n}\right), \mathbb{E}\left(\widetilde{Z}_{n} \mid \mathcal{F}_{n-1}\right)=Z_{n-1} .
$$

Proposition 3.1 On the set $\{n \leq \tau\}, \widetilde{Z}_{n}$ and $Z_{n-1}$ are positive. On the set $\{n>\tau\}, \widetilde{Z}_{n}$ and $Z_{n-1}$ are (strictly) smaller than 1 .

Proof. The first assertion is obtained from the two following equalities:

$$
\begin{aligned}
\mathbb{E}\left(\mathbb{1}_{\{n \leq \tau\}} \mathbb{1}_{\left\{Z_{n-1}=0\right\}}\right) & =\mathbb{E}\left(\mathbb{P}\left(n \leq \tau \mid \mathcal{F}_{n-1}\right) \mathbb{1}_{\left\{Z_{n-1}=0\right\}}\right)=\mathbb{E}\left(Z_{n-1} \mathbb{1}_{\left\{Z_{n-1}=0\right\}}\right)=0, \\
\mathbb{E}\left(\mathbb{1}_{\{n \leq \tau\}} \mathbb{1}_{\left\{\widetilde{Z}_{n}=0\right\}}\right) & =\mathbb{E}\left(\mathbb{P}\left(n \leq \tau \mid \mathcal{F}_{n}\right) \mathbb{1}_{\left\{\widetilde{Z}_{n}=0\right\}}\right)=\mathbb{E}\left(\widetilde{Z}_{n} \mathbb{1}_{\left\{\widetilde{Z}_{n}=0\right\}}\right)=0 .
\end{aligned}
$$

The second assertion is left to the reader.

We give a useful lemma. The proof of a) is standard, the proof of b) can be found in Aksamit et al. [4] for continuous time. For the ease of the reader, we recall these proofs. 
Lemma 3.2 One has, for any random time $\tau$,

a) if the random variable $Y$ is integrable

$$
\mathbb{E}\left(Y \mid \mathcal{G}_{n}\right) \mathbb{1}_{\{\tau>n\}}=\mathbb{1}_{\{\tau>n\}} \frac{\mathbb{E}\left(Y \mathbb{1}_{\{n<\tau\}} \mid \mathcal{F}_{n}\right)}{Z_{n}}, \forall n \geq 0 .
$$

b) for $Y_{n}$ integrable and $\mathcal{F}_{n}$-measurable

$$
\begin{aligned}
\mathbb{E}\left(Y_{n} \mid \mathcal{G}_{n-1}\right) \mathbb{1}_{\{\tau \geq n\}} & =\mathbb{1}_{\{\tau \geq n\}} \frac{1}{Z_{n-1}} \mathbb{E}\left(Y_{n} \widetilde{Z}_{n} \mid \mathcal{F}_{n-1}\right), \forall n \geq 1 \\
\mathbb{E}\left(\frac{Y_{n}}{\widetilde{Z}_{n}} \mid \mathcal{G}_{n-1}\right) \mathbb{1}_{\{\tau \geq n\}} & =\mathbb{1}_{\{\tau \geq n\}} \frac{1}{Z_{n-1}} \mathbb{E}\left(Y_{n} \mathbb{1}_{\left\{\widetilde{Z}_{n}>0\right\}} \mid \mathcal{F}_{n-1}\right), \forall n \geq 1 .
\end{aligned}
$$

Proof.

a) Taking $Y_{n}=\mathbb{E}\left(Y \mid \mathcal{G}_{n}\right)$ in (4), and taking expectation w.r.t. $\mathcal{F}_{n}$ we obtain

$$
\mathbb{E}\left(Y \mathbb{1}_{\{n<\tau\}} \mid \mathcal{F}_{n}\right)=\mathbb{E}\left(\mathbb{1}_{\{n<\tau\}} \mid \mathcal{F}_{n}\right) y_{n}=Z_{n} y_{n} .
$$

b) Only the second equality requires a proof. For $n \geq 1$, we have

$$
\begin{aligned}
\mathbb{E}\left(\frac{Y_{n}}{\widetilde{Z}_{n}} \mathbb{1}_{\{\tau \geq n\}} \mid \mathcal{G}_{n-1}\right) & =\mathbb{1}_{\{\tau \geq n\}} \frac{1}{Z_{n-1}} \mathbb{E}\left(Y_{n} \frac{1}{\widetilde{Z}_{n}} \mathbb{1}_{\{\tau \geq n\}} \mid \mathcal{F}_{n-1}\right) \\
& =\mathbb{1}_{\{\tau \geq n\}} \frac{1}{Z_{n-1}} \mathbb{E}\left(Y_{n} \frac{1}{\widetilde{Z}_{n}} \mathbb{1}_{\{\tau \geq n\}} \mathbb{1}_{\left\{\widetilde{Z}_{n}>0\right\}} \mid \mathcal{F}_{n-1}\right)=\mathbb{1}_{\{\tau \geq n\}} \frac{1}{Z_{n-1}} \mathbb{E}\left(Y_{n} \mathbb{1}_{\left\{\widetilde{Z}_{n}>0\right\}} \mid \mathcal{F}_{n-1}\right) .
\end{aligned}
$$

Lemma 3.3 Let $H_{n}=\mathbb{1}_{\{\tau \leq n\}}, n \geq 0$, and $\Lambda$ be the $\mathbb{F}$-predictable process defined as

$$
\Lambda_{0}=0, \Delta \Lambda_{n}:=\frac{\Delta A_{n}}{Z_{n-1}} \mathbb{1}_{\left\{Z_{n-1}>0\right\}}, n \geq 1 .
$$

The process $N$ defined as

$$
N_{n}:=H_{n}-\Lambda_{n \wedge \tau}=H_{n}-\sum_{k=1}^{n \wedge \tau} \lambda_{k}, n \geq 0
$$

where $\lambda_{n}:=\Delta \Lambda_{n}$ is a $\mathbb{G}$-martingale.

Proof. It suffices to find the Doob decomposition of the $\mathbb{G}$-semimartingale $H$. The predictable part of this decomposition is $K$ with

$$
\begin{aligned}
\Delta K_{n} & \left.=\mathbb{E}\left(\Delta H_{n} \mid \mathcal{G}_{n-1}\right)=\mathbb{1}_{\{\tau \leq n-1}\right\} 0+\mathbb{1}_{\{\tau>n-1\}} \frac{\mathbb{E}\left(\Delta H_{n} \mid \mathcal{F}_{n-1}\right)}{Z_{n-1}} \\
& =\mathbb{1}_{\{\tau \geq n\}} \frac{\mathbb{E}\left(Z_{n-1}-Z_{n} \mid \mathcal{F}_{n-1}\right)}{Z_{n-1}}=\mathbb{1}_{\{\tau \geq n\}} \frac{A_{n}-A_{n-1}}{Z_{n-1}}, n \geq 1 .
\end{aligned}
$$

We conclude, noting that $Z_{n-1}>0$ on $\{1 \leq n \leq \tau\}$, so that, on $\{n \leq \tau\}$, one has $\Delta K_{n}=$ $\lambda_{n}$ where $\lambda_{n}=\frac{\Delta A_{n}}{Z_{n-1}} \mathbb{1}_{\left\{Z_{n-1}>0\right\}}$ is $\mathbb{F}$-predictable. Note for future use that $0 \leq \lambda_{n} \leq \mathbb{E}(1-$ $\left.\frac{Z_{n}}{Z_{n-1}} \mid \mathcal{F}_{n-1}\right) \leq 1$.

Proposition 3.4 Suppose $Z$ positive. The multiplicative decomposition of $Z$ is given by $Z_{n}=$ $N_{n}^{Z} \mathcal{E}(-\Lambda)_{n}, n \geq 0$ where $N^{Z}$ is an $\mathbb{F}$-martingale and $\Lambda$ is defined in Lemma 3.3.

Proof. We have seen that there exist an $\mathbb{F}$-martingale $N^{Z}$ and an $\mathbb{F}$-predictable process $K^{Z}$ such that $Z=N^{Z} K^{Z}$ with

$$
K_{n}^{Z}=\prod_{k=1}^{n}\left[\frac{Z_{k-1}-\mathbb{E}\left(Z_{k} \mid \mathcal{F}_{k-1}\right)}{Z_{k-1}}+1\right], \quad \forall n \geq 1 .
$$

$>$ From Lemma 3.3 and the positivity of $Z$, we have

$$
\Delta \Lambda_{n}=\frac{Z_{n-1}-\mathbb{E}\left(Z_{n} \mid \mathcal{F}_{n-1}\right)}{Z_{n-1}}, \quad \forall n \geq 1,
$$

then by definition of the exponential process, we get that $K^{Z}=\mathcal{E}(-\Lambda)$. 


\subsubsection{Properties}

Lemma 3.5 If $\widetilde{Z}$ is predictable, and $N$ is defined in (6), then $\mathbb{E}\left(N_{n} \mid \mathcal{F}_{n}\right)-N_{0}=M_{0}-M_{n}$ for all $n \geq 0$, where $M$ is the martingale part in the Doob decomposition of $Z$.

Proof. By definition of $N$, we have that, for $n \geq 0$,

$$
\begin{aligned}
\mathbb{E}\left(\Delta N_{n} \mid \mathcal{F}_{n}\right) & =\mathbb{E}\left(\mathbb{1}_{\{\tau \leq n\}}-\mathbb{1}_{\{\tau \leq n-1\}}-\lambda_{n} \mathbb{1}_{\{\tau \geq n\}} \mid \mathcal{F}_{n}\right) \\
& =\mathbb{E}\left(-\mathbb{1}_{\{\tau>n\}}+\mathbb{1}_{\{\tau \geq n\}} \mid \mathcal{F}_{n}\right)-\lambda_{n} \mathbb{E}\left(\mathbb{1}_{\{\tau \geq n\}} \mid \mathcal{F}_{n}\right) \\
& =-Z_{n}+\widetilde{Z}_{n}-\lambda_{n} \widetilde{Z}_{n}=-\Delta Z_{n}-\lambda_{n} Z_{n-1} .
\end{aligned}
$$

Finally, using that $\Delta Z_{n}+\Delta A_{n}=\Delta M_{n}$ and $\lambda_{n}=\mathbb{1}_{\left\{Z_{n-1}>0\right\}} \frac{\Delta A_{n}}{Z_{n-1}}$ which implies

$$
\lambda_{n} Z_{n-1}=\lambda_{n} Z_{n-1} \mathbb{1}_{\left\{Z_{n-1}>0\right\}}+\lambda_{n} Z_{n-1} \mathbb{1}_{\left\{Z_{n-1}=0\right\}}=\Delta A_{n},
$$

and we get $\mathbb{E}\left(\Delta N_{n} \mid \mathcal{F}_{n}\right)=-\Delta M_{n}$.

We denote by $H^{o}$ the increasing integrable $\mathbb{F}$-adapted process ${ }^{2}$

$$
H_{n}^{o}:=\sum_{k=0}^{n} \mathbb{E}\left(\Delta H_{k} \mid \mathcal{F}_{k}\right)=\sum_{k=0}^{n} \mathbb{P}\left(\tau=k \mid \mathcal{F}_{k}\right), \quad \forall n \geq 0,
$$

which satisfies

$$
\mathbb{E}\left(Y_{\tau} \mathbb{1}_{\{\tau<\infty\}}\right)=\mathbb{E}\left(\sum_{n=0}^{\infty} Y_{n} \Delta H_{n}\right)=\mathbb{E}\left(\sum_{n=0}^{\infty} Y_{n} \mathbb{E}\left(\Delta H_{n} \mid \mathcal{F}_{n}\right)\right)=\mathbb{E}\left(\sum_{n=0}^{\infty} Y_{n} \Delta H_{n}^{o}\right)
$$

for any $\mathbb{F}$-adapted bounded process $Y$. We define $H_{\infty}^{o}:=H_{\infty-}^{o}+\mathbb{P}\left(\tau=\infty \mid \mathcal{F}_{\infty}\right)$ where $H_{\infty-}^{o}=\lim _{n \rightarrow \infty} H_{n}^{o}:=\sum_{k=0}^{\infty} \mathbb{P}\left(\tau=k \mid \mathcal{F}_{k}\right)$. Note that $\Delta \widetilde{A}_{n}=H_{n-1}^{o}-H_{n-2}^{o}$, and since $\widetilde{A}_{1}=H_{0}^{o}$ we have $\widetilde{A}_{n}=H_{n-1}^{o}$, hence

$$
Z_{n}+H_{n}^{o}=Z_{n}+\Delta H_{n}^{o}+H_{n-1}^{o}=\widetilde{Z}_{n}+\widetilde{A}_{n}=\widetilde{M}_{n} .
$$

Furthermore, since $\lim _{n \rightarrow \infty} Z_{n}=\mathbb{1}_{\{\tau=\infty\}}$, and $\mathbb{E}\left(H_{\infty-}^{o}\right)=\lim \mathbb{E}\left(H_{n}^{0}\right) \leq 1$, one has

$$
\widetilde{M}_{n}=Z_{n}+H_{n}^{o}=\mathbb{E}\left(\mathbb{1}_{\{\tau=\infty\}}+H_{\infty-}^{o} \mid \mathcal{F}_{n}\right) .
$$

Lemma 3.6 Let $J:=H-\frac{1}{\widetilde{Z}} \mathbb{1}_{[0, \tau]} \cdot H^{o}$. Then, for any integrable $\mathbb{F}$-adapted process $Y$, the process $Y . J$ is a $\mathbb{G}$-martingale. In particular, $J$ is a $\mathbb{G}$-martingale.

Proof. From $\Delta J_{n}=\mathbb{1}_{\{\tau=n\}}-\frac{1}{\widetilde{Z}_{n}} \mathbb{1}_{\{\tau \geq n\}} \mathbb{P}\left(\tau=n \mid \mathcal{F}_{n}\right), n \geq 1$, one has $\Delta J_{n} \mathbb{1}_{\{\tau<n\}}=0$ and, from Lemma $3.2(5)$,

$$
\begin{aligned}
\mathbb{E}\left(Y_{n} \Delta J_{n} \mid \mathcal{G}_{n-1}\right) & =\mathbb{1}_{\{\tau>n-1\}} \frac{1}{Z_{n-1}} \mathbb{E}\left(Y_{n} \Delta J_{n} \mathbb{1}_{\{\tau>n-1\}} \mid \mathcal{F}_{n-1}\right)+\mathbb{E}\left(Y_{n} \Delta J_{n} \mathbb{1}_{\{\tau<n\}} \mid \mathcal{G}_{n-1}\right) \\
& =\mathbb{1}_{\{\tau>n-1\}} \frac{1}{Z_{n-1}} \mathbb{E}\left(Y_{n}\left(\mathbb{P}\left(\tau=n \mid \mathcal{F}_{n}\right)-\frac{1}{\widetilde{Z}_{n}} \mathbb{P}\left(\tau=n \mid \mathcal{F}_{n}\right) \mathbb{P}\left(\tau \geq n \mid \mathcal{F}_{n}\right) \mathbb{1}_{\left\{\widetilde{Z}_{n}>0\right\}}\right) \mid \mathcal{F}_{n-1}\right) \\
& =\mathbb{1}_{\{\tau>n-1\}} \frac{1}{Z_{n-1}} \mathbb{E}\left(Y_{n} \mathbb{P}\left(\tau=n \mid \mathcal{F}_{n}\right)\left(1-\mathbb{1}_{\left\{\widetilde{Z}_{n}>0\right\}}\right) \mid \mathcal{F}_{n-1}\right) \\
& =\mathbb{1}_{\{\tau>n-1\}} \frac{1}{Z_{n-1}} \mathbb{E}\left(Y_{n} \mathbb{P}\left(\tau=n \mid \mathcal{F}_{n}\right) \mathbb{1}_{\left\{\widetilde{Z}_{n=0\}} \mid \mathcal{F}_{n-1}\right)},\right.
\end{aligned}
$$

where the fact that $Y$ is $\mathbb{F}$ adapted has been used in the second equality. It remains to note that, on $\left\{\widetilde{Z}_{n}=0\right\}$, one has $\mathbb{P}\left(\tau=n \mid \mathcal{F}_{n}\right)=0$ to obtain $\mathbb{E}\left(Y_{n} \Delta J_{n} \mid \mathcal{G}_{n-1}\right)=0$.

Note that, if $Y$ is an $\mathbb{F}$-martingale, then, from lemma 1.5, the $\mathbb{G}$-martingale part of $Y$ is orthogonal to $J$. This result is similar to the one obtained by Choulli et al. [6].

\footnotetext{
${ }^{2}$ In fact, $H^{o}$ is the $\mathbb{F}$-dual optional projection of $H$, and many proofs are consequences or properties of dual optional projections. For the ease of the reader, we give the proofs of all the results.
} 


\subsubsection{Immersion in progressive enlargement}

We recall that $\mathbb{F}$ is immersed in $\mathbb{G}$ (we shall write $\mathbb{F} \hookrightarrow \mathbb{G}$ ) if any $\mathbb{F}$-martingale is a $\mathbb{G}$-martingale. This is equivalent to $Z_{n}=\mathbb{P}\left(\tau>n \mid \mathcal{F}_{\infty}\right)=\mathbb{P}\left(\tau>n \mid \mathcal{F}_{k}\right)$ for any $k \geq n \geq 0$.

Lemma 3.7 $\mathbb{F}$ is immersed in $\mathbb{G}$ if and only if $\widetilde{Z}$ is predictable and $\widetilde{Z}_{n}=\mathbb{P}\left(\tau \geq n \mid \mathcal{F}_{\infty}\right), n \geq 0$.

Proof. Assume that $\mathbb{F}$ is immersed in $\mathbb{G}$. Then, for $n \geq 0$,

$$
\begin{aligned}
\widetilde{Z}_{n}=\mathbb{P}\left(\tau \geq n \mid \mathcal{F}_{n}\right) & =\mathbb{P}\left(\tau>n-1 \mid \mathcal{F}_{n}\right)=\mathbb{P}\left(\tau>n-1 \mid \mathcal{F}_{n-1}\right)=\mathbb{P}\left(\tau>n-1 \mid \mathcal{F}_{\infty}\right) \\
& =\mathbb{P}\left(\tau \geq n \mid \mathcal{F}_{\infty}\right),
\end{aligned}
$$

where the second and the next to last equality follow from immersion assumption. The equality $\widetilde{Z}_{n}=\mathbb{P}\left(\tau>n-1 \mid \mathcal{F}_{n-1}\right)=Z_{n-1}$ establishes the predictability of $\widetilde{Z}$.

Assume now that $\widetilde{Z}$ is predictable and $\widetilde{Z}_{n}=\mathbb{P}\left(\tau \geq n \mid \mathcal{F}_{\infty}\right)$. Then, $\widetilde{Z}_{n}=\mathbb{P}\left(\tau \geq n \mid \mathcal{F}_{n-1}\right)$ and

$$
\mathbb{P}\left(\tau>n \mid \mathcal{F}_{n}\right)=\mathbb{P}\left(\tau \geq n+1 \mid \mathcal{F}_{n}\right)=\widetilde{Z}_{n+1}=\mathbb{P}\left(\tau>n \mid \mathcal{F}_{\infty}\right) .
$$

The immersion property follows.

Remark 3.8 We will see in the proof of Theorem 3.24 that $\tilde{Z}$ predictable implies that $\tau$ is a pseudo-stopping time, hence $Z$ (and $\widetilde{Z}$ ) is decreasing.

Theorem 3.9 Suppose $\mathbb{F} \hookrightarrow \mathbb{G}$. Then the following assertions are equivalent

(i) $Z$ is $\mathbb{F}$-predictable.

(ii) For any $\mathbb{G}$-predictable process $U$, one has $\mathbb{E}\left(\sum_{k=1}^{n} U_{k} \Delta N_{k} \mid \mathcal{F}_{n}\right)=0, \forall n \geq 1$, in particular $\mathbb{E}\left(\Delta N_{n} \mid \mathcal{F}_{n}\right)=0, \forall n \geq 1$.

(iii) Any $\mathbb{F}$-martingale $X$ is orthogonal to $N$.

Proof. (i) $\Rightarrow$ (ii). By uniqueness of Doob's decomposition and the predictability of $Z, Z_{n}=$ $M_{0}-A_{n}$, hence $\Delta M_{n}=0$.

By Lemma 3.5 and 3.7, we have that

$$
\mathbb{E}\left(\Delta N_{n} \mid \mathcal{F}_{n}\right)=-\Delta M_{n}
$$

and $\mathbb{E}\left(\Delta N_{n} \mid \mathcal{F}_{n}\right)=0$.

For $k \leq n$, let $\bar{U}_{k} \in \mathcal{F}_{k-1}$ be such that $\bar{U}_{k} \mathbb{1}_{\{\tau>k-1\}}=U_{k} \mathbb{1}_{\{\tau>k-1\}}$, then

$$
\mathbb{E}\left(U_{k} \Delta N_{k} \mid \mathcal{F}_{n}\right)=\bar{U}_{k}\left[\mathbb{E}\left(-\mathbb{1}_{\{\tau>k\}}+\mathbb{1}_{\{\tau \geq k\}} \mid \mathcal{F}_{n}\right)-\lambda_{k} \mathbb{E}\left(\mathbb{1}_{\{\tau \geq k\}} \mid \mathcal{F}_{n}\right)\right],
$$

which, using immersion propetry

$$
\begin{aligned}
\mathbb{E}\left(U_{k} \Delta N_{k} \mid \mathcal{F}_{n}\right) & =\bar{U}_{k}\left[\mathbb{E}\left(-\mathbb{1}_{\{\tau>k\}}+\mathbb{1}_{\{\tau \geq k\}} \mid \mathcal{F}_{k}\right)-\lambda_{k} \mathbb{E}\left(\mathbb{1}_{\{\tau \geq k\}} \mid \mathcal{F}_{k}\right)\right] \\
& =\bar{U}_{k} \mathbb{E}\left(\Delta N_{k} \mid \mathcal{F}_{k}\right)=0,
\end{aligned}
$$

taking the sum over all $k \leq n$ we obtain the desired result.

(ii) $\Rightarrow$ (iii). We prove that $\mathbb{E}\left(\Delta X_{n} \Delta N_{n} \mid \mathcal{G}_{n-1}\right)=0$ for all $n \geq 1$.

From the Lemma 3.2, we have that

$$
\mathbb{E}\left(\Delta X_{n} \Delta N_{n} \mid \mathcal{G}_{n-1}\right) \mathbb{1}_{\{\tau \geq n\}}=\frac{1}{Z_{n-1}} \mathbb{E}\left[\Delta X_{n} \mathbb{1}_{\{\tau>n-1\}} \Delta N_{n} \mid \mathcal{F}_{n-1}\right] \mathbb{1}_{\{\tau \geq n\}},
$$

since $\Delta X_{n} \in \mathcal{F}_{n}$ and $\mathbb{1}_{\{\tau>n-1\}} \in \mathcal{G}_{n-1}$ we have, from (ii)

$$
\mathbb{E}\left(\Delta X_{n} \mathbb{1}_{\{\tau>n-1\}} \Delta N_{n} \mid \mathcal{F}_{n-1}\right)=\mathbb{E}\left[\Delta X_{n} \mathbb{E}\left(\mathbb{1}_{\{\tau>n-1\}} \Delta N_{n} \mid \mathcal{F}_{n}\right) \mid \mathcal{F}_{n-1}\right]=0
$$

hence

$$
\mathbb{E}\left(\Delta X_{n} \Delta N_{n} \mid \mathcal{G}_{n-1}\right) \mathbb{1}_{\{\tau \geq n\}}=0 .
$$

On the set $\{\tau<n\}$, using that $\{\tau<n\} \in \mathcal{G}_{n-1}$, we obtain

$$
\mathbb{E}\left(\Delta X_{n} \Delta N_{n} \mid \mathcal{G}_{n-1}\right) \mathbb{1}_{\{\tau<n\}}=\mathbb{E}\left[\Delta X_{n}\left(\mathbb{1}_{\{\tau=n\}}-\lambda_{n} \mathbb{1}_{\{\tau \geq n\}}\right) \mathbb{1}_{\{\tau<n\}} \mid \mathcal{G}_{n-1}\right]=0 .
$$


Finally, we get $\mathbb{E}\left[\Delta\left(X_{n} N_{n}\right) \mid \mathcal{G}_{n-1}\right]=0$.

(iii) $\Rightarrow$ (i). By (iii), we have in the one hand, for $n \geq 1, \mathbb{E}\left(\Delta X_{n} \Delta N_{n} \mid \mathcal{G}_{n-1}\right)=0$, then $\mathbb{E}\left(\Delta X_{n} \Delta N_{n}\right)=0$. In the other hand, $\mathbb{E}\left(\Delta X_{n} \Delta N_{n}\right)=\mathbb{E}\left[\Delta X_{n} \mathbb{E}\left(\Delta N_{n} \mid \mathcal{F}_{n}\right)\right]$, and applying Lemma 3.5, we obtain $\mathbb{E}\left(\Delta N_{n} \Delta X_{n}\right)=-\mathbb{E}\left(\left|\Delta X_{n}\right|^{2}\right)$, which implies $\mathbb{E}\left(\left|\Delta X_{n}\right|^{2}\right)=0$. Therefore $\Delta X_{n}=0$, or equivalently $\mathbb{E}\left(Z_{n} \mid \mathcal{F}_{n-1}\right)=Z_{n}$, which is equivalent to the predictability of $Z$.

Example 3.10 Assume that $\tau=\inf \left\{n: \Gamma_{n} \geq \Theta\right\}$ where $\Gamma$ is an increasing $\mathbb{F}$-adapted process and $\Theta$ is independent from $\mathbb{F}$, with an exponential law. Then, immersion property holds and $Z_{n}=\mathbb{P}\left(\Gamma_{n}>\Theta \mid \mathcal{F}_{n}\right)=e^{-\Gamma_{n}}\left(\right.$ and $\left.\widetilde{Z}_{n}=\mathbb{P}\left(\tau>n-1 \mid \mathcal{F}_{n}\right)=\mathbb{P}\left(\Gamma_{n-1}>\Theta \mid \mathcal{F}_{n}\right)=e^{-\Gamma_{n-1}}=Z_{n-1}\right)$.

If $\Gamma$ is predictable, the Doob decomposition of $Z$ is $Z_{n}=1-A_{n}=1-e^{-\Gamma_{n}}$, and $\Delta \Lambda_{n}=\frac{\Delta A_{n}}{Z_{n-1}}$. Moreover, $Z$ is predictable and assertions of Theorem 3.9 hold.

If $\Gamma$ is not predictable,

$$
\Delta \Lambda_{n}=\frac{\Delta A_{n}}{Z_{n-1}}=\frac{\mathbb{E}\left(-\Delta Z_{n} \mid \mathcal{F}_{n-1}\right)}{Z_{n-1}}=e^{-\Gamma_{n-1}} \frac{1-\mathbb{E}\left(e^{-\Delta \Gamma_{n}} \mid \mathcal{F}_{n-1}\right)}{Z_{n-1}}=1-\mathbb{E}\left(e^{-\Delta \Gamma_{n}} \mid \mathcal{F}_{n-1}\right) .
$$

\subsubsection{Construction of $\tau$ from a given supermartingale}

We now answer the following question. Let $Z$ be a supermartingale on $(\Omega, \mathbb{F}, \mathbb{P})$, valued in $[0,1]$ such that $Z_{\infty}=0$. Is it possible to construct $\tau$ such that $Z$ is its Azéma supermartingale. We mimic the general proof of Song [10]. It is rather obvious that one has to extend the probability space. Let us consider the space $(\Omega \times \mathbb{N}, \mathbb{F} \otimes \mathcal{N}, \mathbb{Q})$ where $\mathbb{N}$ is the set of non-negative integer, $\mathcal{N}$ the associated $\sigma$-algebra and $\mathbb{Q}$ a probability to be constructed so that the identity map $\tau(\omega, n)=n$ satisfies $\mathbb{Q}\left(\tau>n \mid \mathcal{F}_{n}\right)=Z_{n}$ and $\mathbb{Q}$ coincides with $\mathbb{P}$ on $\mathbb{F}$. To do so, we need to construct a family of martingales $M^{k}$ which will represent $\mathbb{Q}\left(\tau \leq k \mid \mathcal{F}_{n}\right)$. The knowledge of these quantities will allow us to characterize $\mathbb{Q}\left(\{\tau \leq k\} \cap F_{n}\right)$ for any $F_{n} \in \mathcal{F}_{n}$, and using Kolmogorov arguments, to construct $\mathbb{Q}$ on the product space. The measure $\mathbb{Q}$ will be a probability, since $\mathbb{Q}\left(\mathbb{R}^{+} \times \Omega\right)=1$. This family must be valued in $[0,1]$, increasing w.r.t. $k$. i.e.,

$$
M_{k}^{k}=1-Z_{k}, M_{n}^{k} \leq M_{n}^{k+1}, \forall k<n
$$

and $\forall n \leq k, M_{n}^{k}=\mathbb{E}\left(M_{k}^{k} \mid \mathcal{F}_{n}\right)$.

We assume that $1>\mathbb{E}\left(Z_{n} \mid \mathcal{F}_{n-1}\right), \forall n \geq 1$. Let $k$ be fixed and define

$$
M_{k}^{k}:=1-Z_{k}, M_{n}^{k}=M_{n-1}^{k} \frac{\mathbb{E}\left(Z_{n} \mid \mathcal{F}_{n-1}\right)-Z_{n}}{1-\mathbb{E}\left(Z_{n} \mid \mathcal{F}_{n-1}\right)}, \forall n>k
$$

It is easy to check that $M^{k}$ is an $\mathbb{F}$-martingale valued in $[0,1]$ such that $M_{n}^{k} \leq M_{n}^{k+1}$ (the supermartingale property of $Z$ being used to obtain $M_{n}^{k} \geq 0$ ).

\subsection{Study before $\tau$}

\subsubsection{Semimartingale decomposition}

Proposition 3.11 Any square integrable $\mathbb{F}$-martingale $X$ stopped at $\tau$ is a $\mathbb{G}$-semimartingale with decomposition

$$
X_{n}^{\tau}=X_{n}^{\mathbb{G}}+\sum_{k=1}^{n \wedge \tau} \frac{1}{Z_{k-1}} \Delta\langle\widetilde{M}, X\rangle_{k}^{\mathbb{F}},
$$

where $X^{\mathbb{G}}$ is a $\mathbb{G}$-martingale (stopped at $\tau$ ). Here, $\widetilde{M}$ is the martingale part of the Doob decomposition of the supermartingale $\widetilde{Z}$.

Proof. We compute the predictable part of the $\mathbb{G}$-semimartingale $X$ on the set $\{0 \leq n<\tau\}$ using Lemma 3.2

$$
\mathbb{1}_{\{\tau>n\}} \mathbb{E}\left(\Delta X_{n+1} \mid \mathcal{G}_{n}\right)=\mathbb{1}_{\{\tau>n\}} \frac{1}{Z_{n}} \mathbb{E}\left(\widetilde{Z}_{n+1} \Delta X_{n+1} \mid \mathcal{F}_{n}\right) .
$$

Using now the Doob decomposition of $\widetilde{Z}$, and the martingale property of $X$, we obtain

$$
\begin{aligned}
\mathbb{E}\left(\widetilde{Z}_{n+1} \Delta X_{n+1} \mid \mathcal{F}_{n}\right) & =\mathbb{E}\left(\left(\widetilde{M}_{n+1}-\widetilde{A}_{n+1}\right) \Delta X_{n+1} \mid \mathcal{F}_{n}\right) \\
& =\mathbb{E}\left(\widetilde{M}_{n+1} \Delta X_{n+1} \mid \mathcal{F}_{n}\right)=\Delta\langle\widetilde{M}, X\rangle_{n+1}^{\mathbb{F}}
\end{aligned}
$$


and finally

$$
\mathbb{1}_{\{\tau>n\}} \mathbb{E}\left(\Delta X_{n+1} \mid \mathcal{G}_{n}\right)=\mathbb{1}_{\{\tau>n\}} \frac{1}{Z_{n}} \Delta\langle\widetilde{M}, X\rangle_{n+1}^{\mathbb{F}}
$$

Comment 3.12 We recall, for the ease of the reader, the Jeulin formula in continuous time: If $\mathbb{G}$ is the progressive enlargement of $\mathbb{F}$ with a random time $\tau$, any $\mathbb{F}$ martingale $X$ stopped at $\tau$ is a $\mathbb{G}$ semimartingale with decomposition

$$
X_{t}^{\tau}=\widehat{X}_{t}+\int_{0}^{t \wedge \tau} \frac{1}{Z_{s-}} d\langle\widetilde{M}, X\rangle_{s}^{\mathbb{F}}
$$

where $Z_{t}=\mathbb{P}\left(\tau>t \mid \mathcal{F}_{t}\right), \quad \widetilde{Z}_{t}=\mathbb{P}\left(\tau \geq t \mid \mathcal{F}_{t}\right)$. Here $\widetilde{Z}=\widetilde{M}-\widetilde{A}$ where $\widetilde{M}$ is an $\mathbb{F}$-martingale and $\widetilde{A}$ is $\mathbb{F}$-predictable. As $\widetilde{Z}$ is not càdlàg, this is not the standard Doob-Meyer decomposition established only for càdlàg supermartingales.

\subsubsection{Arbitrages}

Lemma 3.13 Let $\mathbb{G}^{\tau-}$ be the filtration $\mathbb{G}$ "strictly before $\tau$ ", i.e., $\mathcal{G}_{n}^{\tau-}=\mathcal{G}_{n \wedge(\tau-1)}$. The model $\left(\mathbb{F}, \mathbb{G}^{\tau-}\right)$ is arbitrage free.

Proof. In the case where $X$ is an $\mathbb{F}$-martingale and working in the progressive enlarged filtration, we will find $\psi$ such that on the set $\{1 \leq n<\tau\}$ (strictly before $\tau$ )

$$
\mathbb{1}_{\{n-1 \leq \tau\}} \mathbb{E}\left(\psi_{n} X_{n} \mid \mathcal{G}_{n-1}\right)=\mathbb{1}_{\{n-1 \leq \tau\}} X_{n-1} \mathbb{E}\left(\psi_{n} \mid \mathcal{G}_{n-1}\right)
$$

that is

$$
\mathbb{1}_{\{n-1 \leq \tau\}} \frac{1}{\widetilde{Z}_{n-1}} \mathbb{E}\left(\psi_{n} X_{n} Z_{n} \mid \mathcal{F}_{n-1}\right)=\mathbb{1}_{\{n-1 \leq \tau\}} \frac{X_{n-1}}{\widetilde{Z}_{n-1}} \mathbb{E}\left(\psi_{n} Z_{n} \mid \mathcal{F}_{n-1}\right) .
$$

We are looking for a positive $\mathbb{F}$-adapted process $\psi$, satisfying

$$
\mathbb{E}\left(\psi_{n} X_{n} Z_{n} \mid \mathcal{F}_{n-1}\right)=X_{n-1} \mathbb{E}\left(\psi_{n} Z_{n} \mid \mathcal{F}_{n-1}\right)
$$

The choice $\psi=(1 / Z) \mathbb{1}_{\{Z>0\}}+\mathbb{1}_{\{Z=0\}}$ provides a solution, valid for any martingale $X$.

Theorem 3.14 Assume that $\tau$ is not an $\mathbb{F}$-stopping time and denote by $\mathbb{G}^{\tau}$ the filtration $\mathcal{G}_{n}^{\tau}=$ $\mathcal{G}_{\tau \wedge n}, n \geq 0$. Then, the model $\left(\mathbb{F}, \mathbb{G}^{\tau}\right)$ is arbitrage free if and only, for any $n$, the set $\left\{0=\widetilde{Z}_{n}<\right.$ $\left.Z_{n-1}\right\}$ is empty.

We mean here that, for any $\mathbb{F}$-martingale $X$, the stopped process $X^{\tau}$ admits a deflator. This result was established in Choulli and Deng [7] and is a particular case of the general results obtained in Aksamit et al.[4]. We give here a slightly different proof, by means of the two following propositions.

Proposition 3.15 Assume that for any $n$, the set $\left\{\widetilde{Z}_{n}=0<Z_{n-1}\right\}$ is empty. The process $L=\mathcal{E}(Y)$, where $Y$ is the $\mathbb{G}$-martingale defined by $\Delta Y_{k}=\mathbb{1}_{\{\tau \geq k\}}\left(\frac{Z_{k-1}}{\widetilde{Z}_{k}}-1\right)$ for $k \geq 1$ and $Y_{0}=0$, is a positive $\mathbb{G}$-martingale. If $X$ is an $\mathbb{F}$-martingale, the process $X^{\tau} L$ is a $(\mathbb{G}, \mathbb{P})$ martingale.

Proof. The process $Y$ is a martingale: for $n \geq 1$,

$$
\begin{aligned}
& \mathbb{E}\left(\Delta Y_{n} \mid \mathcal{G}_{n-1}\right)=\mathbb{E}\left(\mathbb{1}_{\{\tau \geq n\}} \frac{Z_{n-1}-\widetilde{Z}_{n}}{\widetilde{Z}_{n}} \mid \mathcal{G}_{n-1}\right)=\mathbb{1}_{\{\tau \geq n\}} \frac{1}{Z_{n-1}} \mathbb{E}\left(\mathbb{1}_{\left\{\widetilde{Z}_{n}>0\right\}}\left(Z_{n-1}-\widetilde{Z}_{n}\right) \mid \mathcal{F}_{n-1}\right) \\
& =\mathbb{1}_{\{\tau \geq n\}} \frac{1}{Z_{n-1}} \mathbb{E}\left(Z_{n-1}-\widetilde{Z}_{n}-\mathbb{1}_{\left\{\widetilde{Z}_{n}=0\right\}}\left(Z_{n-1}-\widetilde{Z}_{n}\right) \mid \mathcal{F}_{n-1}\right) \\
& =\mathbb{1}_{\{\tau \geq n\}} \frac{1}{Z_{n-1}} \mathbb{E}\left(Z_{n-1}-\widetilde{Z}_{n} \mid \mathcal{F}_{n-1}\right)=0,
\end{aligned}
$$

where we have used (5), the fact that $\mathbb{E}\left(\widetilde{Z}_{n} \mid \mathcal{F}_{n-1}\right)=Z_{n-1}$ and that, by assumption $\left\{\widetilde{Z}_{n}=0\right\} \subset$ $\left\{Z_{n-1}=0\right\}$, hence $\mathbb{1}_{\left\{\widetilde{Z}_{n}=0\right\}}\left(Z_{n-1}-\widetilde{Z}_{n}\right)=0$.

Hence $L$ is a martingale. Note that the fact that $\left\{Z_{n-1}=0\right\} \subset\left\{\widetilde{Z}_{n}=0\right\}$ implies that the inclusion $\left\{\widetilde{Z}_{n}=0\right\} \subset\left\{Z_{n-1}=0\right\}$ is equivalent to $\left\{\widetilde{Z}_{n}=0\right\}=\left\{Z_{n-1}=0\right\}$, or to 
$\left\{\widetilde{Z}_{n}=0<Z_{n-1}\right\}$ is empty. On the set $\{\tau \geq k\}$, one has $Z_{k-1}>0$ which implies that $\Delta Y_{k}=\left(\frac{Z_{k-1}}{\widetilde{Z}_{k}}-1\right) \geq-1$, hence $L$ is positive. Furthermore, for $X$ an $\mathbb{F}$-martingale

$$
\begin{aligned}
\mathbb{E}\left(X_{n+1}^{\tau} \frac{L_{n+1}}{L_{n}} \mid \mathcal{G}_{n}\right) & =\mathbb{E}\left(X_{(n+1) \wedge \tau}\left(1+\mathbb{1}_{\{\tau \geq n+1\}} \frac{Z_{n}-\widetilde{Z}_{n+1}}{\widetilde{Z}_{n+1}}\right) \mid \mathcal{G}_{n}\right) \\
& =\mathbb{E}\left(X_{n+1} \mathbb{1}_{\{\tau \geq n+1\}} \frac{Z_{n}}{\widetilde{Z}_{n+1}} \mid \mathcal{G}_{n}\right)+\mathbb{E}\left(X_{\tau} \mathbb{1}_{\{\tau<n+1\}} \mid \mathcal{G}_{n}\right) \\
& =\mathbb{E}\left(X_{n+1} \mathbb{1}_{\{\tau \geq n+1\}} \frac{Z_{n}}{\widetilde{Z}_{n+1}} \mid \mathcal{G}_{n}\right)+X_{\tau} \mathbb{1}_{\{\tau<n+1\}} \\
& =\mathbb{1}_{\{\tau>n\}} \frac{1}{Z_{n}} \mathbb{E}\left(X_{n+1} Z_{n} \mathbb{1}_{\left\{\widetilde{Z}_{n+1}>0\right\}} \mid \mathcal{F}_{n}\right)+X_{\tau} \mathbb{1}_{\{\tau \leq n\}} \\
& =\mathbb{1}_{\{\tau>n\}} \frac{1}{Z_{n}} \mathbb{E}\left(X_{n+1} Z_{n}\left(1-\mathbb{1}_{\left\{\widetilde{Z}_{n+1}=0\right\}}\right) \mid \mathcal{F}_{n}\right)+X_{\tau} \mathbb{1}_{\{\tau \leq n\}} \\
& =\mathbb{1}_{\{\tau>n\}} \frac{1}{Z_{n}} \mathbb{E}\left(X_{n+1} Z_{n} \mid \mathcal{F}_{n}\right)+X_{\tau} \mathbb{1}_{\{\tau \leq n\}}=X_{n \wedge \tau},
\end{aligned}
$$

where we have used that, by assumption, $Z_{n} \mathbb{1}_{\left\{\widetilde{Z}_{n+1}=0\right\}}=0$. Hence the deflator property.

Remark 3.16 In case of immersion, there are no arbitrages (indeed any e.m.m. in $\mathbb{F}$ will be an e.m.m. in $\mathbb{G}$ ). This can be also obtained using the previous result, since, under immersion hypothesis, one has $Z_{n-1}=\widetilde{Z}_{n}$.

Proposition 3.17 If there exists $n \geq 1$ such that the set $\left\{0=\widetilde{Z}_{n}<Z_{n-1}\right\}$ is not empty, and if $\tau$ is not an $\mathbb{F}$-stopping time, there exists an $\mathbb{F}$-martingale $X$ such that $X^{\tau}$ is a $\mathbb{G}$-adapted increasing process with $X_{0}^{\tau}=1, \mathbb{P}\left(X_{\tau}^{\tau}>1\right)>0$. Hence, the model $\left(\mathbb{F}, \mathbb{G}^{\tau}\right)$ is not arbitrage free.

Proof. The proof is the discrete version of Acciaio et al. [1]. Let $\vartheta=\inf \left\{n: 0=\widetilde{Z}_{n}<Z_{n-1}\right\}$. The random time $\vartheta$ is an $\mathbb{F}$-stopping time satisfying $\tau \leq \vartheta$ and $\mathbb{P}(\tau<\vartheta)>0$. Let $I_{n}=\mathbb{1}_{\{\vartheta \leq n\}}$ and denote by $D$ the $\mathbb{F}$-predictable process part of the Doob decomposition of $I$. One has $D_{0}=0$ and $\Delta D_{n}=\mathbb{P}\left(\vartheta=n \mid \mathcal{F}_{n-1}\right)$. We introduce the $\mathbb{F}$-predictable increasing process $U$ setting $U_{n}=\frac{1}{\mathcal{E}(-D)_{n}}$. Then,

$$
\Delta U_{n}=\frac{1}{\mathcal{E}(-D)_{n-1}}\left(\frac{1}{1-\Delta D_{n}}-1\right)=\frac{1}{\mathcal{E}(-D)_{n-1}} \frac{\Delta D_{n}}{1-\Delta D_{n}}=U_{n} \Delta D_{n}
$$

We consider the process $X=U K$, where $K=1-I$,

$$
\Delta X_{n}=-U_{n} \Delta I_{n}+K_{n-1} \Delta U_{n}=-U_{n}\left(\Delta I_{n}-K_{n-1} \Delta D_{n}\right)
$$

and

$$
\begin{aligned}
\mathbb{E}\left(\Delta X_{n} \mid \mathcal{F}_{n-1}\right) & =-U_{n} \mathbb{E}\left(\Delta I_{n}-K_{n-1} \Delta D_{n} \mid F_{n-1}\right)=U_{n}\left(\mathbb{P}\left(\vartheta=n \mid \mathcal{F}_{n-1}\right)-K_{n-1} \mathbb{P}\left(\Delta D_{n} \mid F_{n-1}\right)\right) \\
& =U_{n} K_{n-1}\left(\mathbb{P}\left(\vartheta=n \mid \mathcal{F}_{n-1}\right)-\mathbb{P}\left(\Delta D_{n} \mid F_{n-1}\right)\right)=0,
\end{aligned}
$$

where we have used that $K_{n-1} \mathbb{P}\left(\vartheta=n \mid \mathcal{F}_{n-1}\right)=\mathbb{E}\left(K_{n-1} \mathbb{1}_{\vartheta=n} \mid \mathcal{F}_{n-1}\right)=\mathbb{P}\left(\vartheta=n \mid \mathcal{F}_{n-1}\right)$. Hence $X$ is an $\mathbb{F}$-martingale.

We now prove that $X_{\tau} \geq 1$ and $\mathbb{P}\left(X_{\tau}>1\right)>0$, equivalently that $D_{\tau} \geq 0$ and $\mathbb{P}\left(D_{\tau}>0\right)>0$. For that, we compute

$$
\begin{aligned}
\mathbb{E}\left(D_{\tau} \mathbb{1}_{\tau<\infty}\right) & =\sum_{n=0}^{\infty} \mathbb{E}\left(D_{n} \mathbb{1}_{\{\tau=n\}}\right)=\sum_{n=0}^{\infty} \mathbb{E}\left(D_{n} \mathbb{P}\left(\tau=n \mid \mathcal{F}_{n}\right)\right) \\
& =\sum_{n=1}^{\infty} \mathbb{E}\left(D_{n} \mathbb{P}\left(\tau>n \mid \mathcal{F}_{n}\right)\right)-\sum_{n=1}^{\infty} \mathbb{E}\left(D_{n} \mathbb{P}\left(\tau>n-1 \mid \mathcal{F}_{n}\right)\right)+D_{0} \mathbb{P}(\tau=0)
\end{aligned}
$$

Since $D$ is predictable

$$
\begin{aligned}
\mathbb{E}\left(D_{\tau} \mathbb{1}_{\tau<\infty}\right) & =\sum_{n=1}^{\infty} \mathbb{E}\left(D_{n} \mathbb{P}\left(\tau>n \mid \mathcal{F}_{n}\right)\right)-\sum_{n=1}^{\infty} \mathbb{E}\left(D_{n} \mathbb{P}\left(\tau>n-1 \mid \mathcal{F}_{n-1}\right)\right)=-\sum_{n=1}^{\infty} \mathbb{E}\left(D_{n} \Delta Z_{n}\right) \\
& =\mathbb{E}\left(\sum_{n=1}^{\infty} Z_{n-1} \Delta D_{n}\right)=\mathbb{E}\left(Z_{\vartheta-1} \mathbb{1}_{\vartheta<\infty}\right)>0,
\end{aligned}
$$

where, in the last inequality, we used that $\tau \leq \vartheta$ and $\mathbb{P}(\tau=\vartheta)<1$. The process $X^{\tau}$ is then an increasing process and can not be turned in a martingale by change of probability. 


\section{$3.3 \quad$ After $\tau$}

As we mentioned at the beginning, any $\mathbb{F}$-martingale is a $\mathbb{G}$-semimartingale (which is not the case in continuous time). In a progressive enlargement of filtration with a random time valued in $\mathbb{N}$, one can give the decomposition formula. We start with the general case, then we study the particular case where $\tau$ is honest, to provide comparison with the classical results.

\subsubsection{General case}

Mixing the results obtained in initial enlargement and progressive enlargement before $\tau$, for any $\mathbb{F}$-martingale $X$

$$
X_{n}=X_{n}^{\mathbb{G}}+\sum_{k=0}^{n \wedge \tau} \frac{1}{Z_{k-1}} \Delta\langle\widetilde{M}, X\rangle_{k}+\sum_{k=\tau}^{n} \frac{\left.\Delta\langle X, p(u)\rangle_{k}\right|_{u=\tau}}{p_{k-1}(\tau)} .
$$

where $X^{\mathbb{G}}$ is a $\mathbb{G}$-martingale.

\subsubsection{Honest times}

In continuous time, strong conditions are needed to keep the semimartingale property after $\tau$, here it is no more the case. However, we now consider the case where $\tau$ is honest (and valued in $\mathbb{N}$ ). We recall the definition (see Barlow [5]) and some of the main properties.

A random time is honest, if, for any $n \geq 0$, there exists an $\mathcal{F}_{n}$-measurable random variable $\tau(n)$ such that

$$
\mathbb{1}_{\{\tau \leq n\}} \tau=\mathbb{1}_{\{\tau \leq n\}} \tau(n) .
$$

Remark 3.18 Following Jeulin, $\tau$ is honest if there exists an $\mathcal{F}_{n}$ measurable random variable $\widehat{\tau}(n)$, such that

$$
\mathbb{1}_{\{\tau<n\}} \tau=\mathbb{1}_{\{\tau<n\}} \widehat{\tau}(n) .
$$

The two definitions are equivalent. Indeed, starting with the equality (11), one can define $\tau(n)=\widehat{\tau}(n) \wedge n$; then on $\{\tau=n\}, \tau(n)=n$ and $\mathbb{1}_{\{\tau \leq n\}} \tau=\mathbb{1}_{\{\tau \leq n\}} \tau(n)$.

It follows that any $\mathbb{G}$-predictable process $V$ can be written as $V_{n}=V_{n}^{b} \mathbb{1}_{\{n \leq \tau\}}+V_{n}^{a} \mathbb{1}_{\{\tau<n\}}$ where $V^{a}, V^{b}$ are $\mathbb{F}$-predictable processes.

Lemma 3.19 If $\tau$ is honest, $Z_{n}=\widetilde{Z}_{n}$ on the set $\{n>\tau\}$ and $\widetilde{Z}_{\tau}=1$.

If $\widetilde{Z}_{\tau}=1$, then $\tau$ is honest.

Proof. For any $n \geq 0$,

$$
\begin{aligned}
\mathbb{P}\left(\tau=n \mid \mathcal{F}_{n}\right) \mathbb{1}_{\{n>\tau\}} & =\mathbb{P}\left(\tau=n \mid \mathcal{F}_{n}\right) \mathbb{1}_{\{n>\tau\}} \mathbb{1}_{\{n>\tau(n)\}}=\mathbb{E}\left(\mathbb{1}_{\{\tau=n\}} \mathbb{1}_{\{n>\tau(n)\}} \mid \mathcal{F}_{n}\right) \mathbb{1}_{\{n>\tau\}} \\
& =\mathbb{E}\left(\mathbb{1}_{\{\tau=n\}} \mathbb{1}_{\{n>\tau(n)\}} \mathbb{1}_{\{n>\tau\}} \mid \mathcal{F}_{n}\right) \mathbb{1}_{\{n>\tau\}}=0 .
\end{aligned}
$$

It follows that $Z_{n} \mathbb{1}_{\{\tau<n\}}=\widetilde{Z}_{n} \mathbb{1}_{\{\tau<n\}}$. Furthermore,

$$
\begin{aligned}
\widetilde{Z}_{n} \mathbb{1}_{\{\tau=n\}} & =\mathbb{1}_{\{\tau=n\}} \mathbb{P}\left(\tau \geq n \mid \mathcal{F}_{n}\right)=\mathbb{1}_{\{\tau=n\}} \mathbb{1}_{\{\tau(n)=n\}} \mathbb{P}\left(\tau \geq n \mid \mathcal{F}_{n}\right) \\
& =\mathbb{1}_{\{\tau=n\}} \mathbb{E}\left(\mathbb{1}_{\{\tau(n)=n\}} \mathbb{1}_{\{\tau \geq n\}} \mid \mathcal{F}_{n}\right)=\mathbb{1}_{\{\tau=n\}}
\end{aligned}
$$

which implies $\widetilde{Z}_{\tau}=1$.

If $\widetilde{Z}_{\tau}=1$, let $\ell(n)=\sup \left\{k \leq n: \widetilde{Z}_{k}=1\right\}$. Then, for any $n \geq 0$, one has $\tau=\ell(n)$ on the set $\{\tau \leq n\}$, and $\tau$ is honest.

Proposition 3.20 Let $\tau$ be an honest time and $X$ an $\mathbb{F}$-martingale. Then,

$$
X_{n}=X_{n}^{\mathbb{G}}+\sum_{k=0}^{n \wedge \tau} \frac{1}{Z_{k-1}} \Delta\langle\widetilde{M}, X\rangle_{k}-\sum_{k=\tau+1}^{n} \frac{1}{1-Z_{k-1}} \Delta\langle\widetilde{M}, X\rangle_{k}
$$

where $X^{\mathbb{G}}$ is a $\mathbb{G}$-martingale. 
Proof. Let $X=M^{\mathbb{G}}+V^{\mathbb{G}}$ be the $\mathbb{G}$-semimartingale decomposition of $X$. Let $n \geq 0$ be fixed. From the property of honest times, there exists $\widetilde{V}$, an $\mathbb{F}$-predictable process, such that

$$
V_{n}^{\mathbb{G}} \mathbb{1}_{\{\tau \leq n\}}=\widetilde{V}_{n} \mathbb{1}_{\{\tau \leq n\}} .
$$

Then,

$$
\begin{aligned}
\mathbb{1}_{\{\tau \leq n\}}\left(V_{n+1}^{\mathbb{G}}-V_{n}^{\mathbb{G}}\right) & =\mathbb{1}_{\{\tau \leq n\}}\left(\widetilde{V}_{n+1}-\widetilde{V}_{n}\right)=\mathbb{1}_{\{\tau \leq n\}} \mathbb{E}\left(X_{n+1}-X_{n} \mid \mathcal{G}_{n}\right) \\
& =\mathbb{E}\left(\mathbb{1}_{\{\tau \leq n\}}\left(X_{n+1}-X_{n}\right) \mid \mathcal{G}_{n}\right) .
\end{aligned}
$$

We now take the conditional expectation w.r.t. $\mathcal{F}_{n}$ in (12). Taking into account that $\widetilde{V}$ is $\mathbb{F}$-predictable, and the fact that $\mathbb{F} \subset \mathbb{G}$, we get

$$
\begin{aligned}
\mathbb{E}\left(\mathbb{1}_{\{\tau \leq n\}} \mid \mathcal{F}_{n}\right)\left(\widetilde{V}_{n+1}-\widetilde{V}_{n}\right) & =\mathbb{E}\left(\mathbb{1}_{\{\tau \leq n\}}\left(X_{n+1}-X_{n}\right) \mid \mathcal{F}_{n}\right) \\
& =\mathbb{E}\left(\mathbb{E}\left(\mathbb{1}_{\{\tau \leq n\}} \mid \mathcal{F}_{n+1}\right)\left(X_{n+1}-X_{n}\right) \mid \mathcal{F}_{n}\right) .
\end{aligned}
$$

Now, using the fact that

$$
\begin{aligned}
\mathbb{E}\left(\mathbb{1}_{\{\tau \leq n\}} \mid \mathcal{F}_{n}\right) & =1-\mathbb{E}\left(\mathbb{1}_{\{\tau>n\}} \mid \mathcal{F}_{n}\right)=1-Z_{n} \\
\mathbb{E}\left(\mathbb{1}_{\{\tau \leq n\}} \mid \mathcal{F}_{n+1}\right) & =1-\mathbb{E}\left(\mathbb{1}_{\{\tau>n\}} \mid \mathcal{F}_{n+1}\right)=1-\mathbb{E}\left(\mathbb{1}_{\{\tau \geq n+1\}} \mid \mathcal{F}_{n+1}\right)=1-\widetilde{Z}_{n+1}
\end{aligned}
$$

and that $X$ is an $\mathbb{F}$-martingale, we obtain, on the set $\{\tau \leq n\}$

$$
\left(1-Z_{n}\right)\left(\widetilde{V}_{n+1}-\widetilde{V}_{n}\right)=-\mathbb{E}\left(\widetilde{Z}_{n+1}\left(X_{n+1}-X_{n}\right) \mid \mathcal{F}_{n}\right)=-\langle\widetilde{M}, X\rangle_{n}
$$

Remark 3.21 It seems important to note that the Doob decomposition of $Z$ is not needed. As we have seen, one can write an optional decomposition of $Z$ as $Z=\widetilde{M}-H^{o}$. This "explains" why, in continuous time, such an optional decomposition of $Z$ is required.

Comment 3.22 We recover the Jeulin's formula for honest times. We recall, for the ease of the reader, the Jeulin formula in continuous time:

$$
X_{n}=X_{n}^{\mathbb{G}}+\int_{0}^{n \wedge \tau} \frac{1}{Z_{s-}} \Delta\langle\widetilde{M}, X\rangle_{s}-\int_{\tau}^{n} \frac{1}{1-Z_{s-}} d\langle\widetilde{M}, X\rangle_{s} .
$$

Comment 3.23 Let $\tau$ an honest time. We have obtained a formula using Jacod's hypothesis in (2.2). In continuous time, one can show that honest times do not satisfy equivalence Jacod's hypothesis. The goal here is to check that the decompositions obtained in (9) and the one for honest times are the same. We proceed as in Aksamit [2]. Let $n \geq 1$ be fixed. On $\tau<n$, we have $\tau=\tau(n-1)$ where $\tau(n-1) \in \mathcal{F}_{n-1} \subset \mathcal{F}_{n}$. We now restrict our attention to $k<n$. On the one hand,

$$
\begin{aligned}
\mathbb{1}_{\{\tau=k\}}\left(1-Z_{n-1}\right) & =\mathbb{1}_{\{\tau=k=\tau(n-1)\}} \mathbb{P}\left(\tau \leq n-1 \mid \mathcal{F}_{n-1}\right)=\mathbb{1}_{\{\tau=k\}} \mathbb{E}\left(\mathbb{1}_{\{\tau(n-1)=k\}} \mathbb{1}_{\{\tau \leq n-1\}} \mid \mathcal{F}_{n-1}\right) \\
& =\mathbb{1}_{\{\tau=k\}} \mathbb{E}\left(\mathbb{1}_{\{\tau(n-1)=k\}} \mathbb{1}_{\{\tau=k\}} \mid \mathcal{F}_{n-1}\right)=\mathbb{1}_{\{\tau=k\}} \mathbb{E}\left(\mathbb{1}_{\{\tau=k\}} \mid \mathcal{F}_{n-1}\right)=\mathbb{1}_{\{\tau=k\}} p_{n-1}^{k}
\end{aligned}
$$

On the other hand

$$
\begin{aligned}
\mathbb{1}_{\{\tau=k\}} \mathbb{E}\left(\widetilde{M}_{n} \Delta X_{n} \mid \mathcal{F}_{n-1}\right) & =-\mathbb{1}_{\{\tau=k\}} \mathbb{E}\left(\left(1-\widetilde{M}_{n}\right) \Delta X_{n} \mid \mathcal{F}_{n-1}\right)=-\mathbb{1}_{\{\tau=k=\tau(n-1)\}} \mathbb{E}\left(\left(1-\widetilde{Z}_{n}\right) \Delta X_{n} \mid \mathcal{F}_{n-1}\right) \\
& =-\mathbb{1}_{\{\tau=k\}} \mathbb{E}\left(\mathbb{1}_{\{k=\tau(n-1)\}} \mathbb{1}_{\{\tau<n\}} \Delta X_{n} \mid \mathcal{F}_{n-1}\right) \\
& =-\mathbb{1}_{\{\tau=k\}} \mathbb{E}\left(\left(\mathbb{E}\left(\mathbb{1}_{\{k=\tau\}} \mid \mathcal{F}_{n}\right) \Delta X_{n} \mid \mathcal{F}_{n-1}\right)=-\mathbb{1}_{\{\tau=k\}} \mathbb{E}\left(p_{n}^{k} \Delta X_{n} \mid \mathcal{F}_{n-1}\right) .\right.
\end{aligned}
$$

\subsubsection{Arbitrages before $\tau$}

Let $\tau$ be a bounded honest time which is not an $\mathbb{F}$-stopping time. Assuming the existence of a deflator $L$ implies that $\widetilde{M} L$ is a $\mathbb{G}$ martingale. Since $\tilde{Z}_{\tau}=1$, one has $\widetilde{M}_{\tau} \geq 1$, and $\mathbb{P}\left(\widetilde{M}_{\tau}>1\right)>0$. Therefore, using optional sampling theorem, $1=\mathbb{E}\left(\widetilde{M}_{\tau} L_{\tau}\right)>\mathbb{E}\left(L_{\tau}\right)=1$ yields to a contradiction and to existence of arbitrages.

We now check that the condition given in 3.14 is satisfied. Let $n_{0}$ such that $\mathbb{P}\left(\tau \leq n_{0}\right)=1$ and $\mathbb{P}\left(\tau \leq n_{0}-1\right)<1$. On the set $A:=\left\{\tau \leq n_{0}-1<n_{0}\right\}$, the honesty of $\tau$ implies that $\widetilde{Z}_{n_{0}}=Z_{n_{0}}=0$. We prove that $\mathbb{P}\left(Z_{n_{0}-1} \mathbb{1}_{A}\right)>0$. The equality $Z_{n_{0}-1} \mathbb{1}_{A}=0$ implies 
that $Z_{n_{0}-1} \mathbb{E}\left(\mathbb{1}_{\left\{\tau \leq n_{0}-1\right\}} \mid \mathcal{H}_{n_{0}-1}\right)=0=Z_{n_{0}-1}\left(1-Z_{n_{0}-1}\right)$, hence $\mathbb{E}\left(Z_{n_{0}-1}\right)=\mathbb{E}\left(Z_{n_{0}-1}^{2}\right)$ which, using the fact that $\mathbb{E}\left(Z_{n_{0}-1}^{2}\right) \leq\left(\mathbb{E}\left(Z_{n_{0}-1}\right)\right)^{2}$ implies that $\mathbb{E}\left(Z_{n_{0}-1}\right)=0$ or $\mathbb{E}\left(Z_{n_{0}-1}\right)=1$, i.e., $Z_{n_{0}-1} \equiv 0$ or $Z_{n_{0}-1} \equiv 1$. The first equality would imply that $\tau=n_{0}$, the second equality that $\tau \leq n_{0}$, a.s., hence a contradiction.

We refer to Choulli and Deng [7] for a necessary and sufficient condition to avoid arbitrages after $\tau$.

\subsection{Pseudo-stopping times}

We end the study of progressive enlargement with a specific class of random times. We assume that $\mathcal{F}_{0}$ is trivial. We recall that a random time $\tau$ is an $\mathbb{F}$-pseudo stopping time if $\mathbb{E}\left(X_{\tau}\right)=\mathbb{E}\left(X_{0}\right)$ for any bounded $\mathbb{F}$-martingale $X$ (see [8]).

Theorem 3.24 The following statements are equivalent:

(i) $\tau$ is an $\mathbb{F}$-pseudo stopping time.

(ii) $H_{\infty-}^{o}=\mathbb{P}\left(\tau<\infty \mid \mathcal{F}_{\infty}\right), H_{\infty}^{o}=1$.

(iii) $\widetilde{M} \equiv 1$.

(iv) $\widetilde{Z}$ is predictable.

(v) Every $\mathbb{F}$-martingale stopped at $\tau$ is a $\mathbb{G}$-martingale.

Proof. The proofs of $((\mathrm{i}) \Leftrightarrow$ (ii) $\Leftrightarrow$ (iii) $\Leftrightarrow(\mathrm{v}))$ are standard.

(i) $\Rightarrow$ (ii) Using that that the bounded martingale $X$ is closed, $\lim _{n \rightarrow \infty} X_{n}=: X_{\infty}$ exists and one can write the integration by parts formula

$$
H_{\infty-}^{o} X_{\infty}=\sum_{n=1}^{\infty} H_{n-1}^{o} \Delta X_{n}+\sum_{n=0}^{\infty} X_{n} \Delta H_{n}^{o} .
$$

Taking expectation, and using the fact that $X$ is an $\mathbb{F}$-martingale, we obtain

$$
\mathbb{E}\left(H_{\infty-}^{o} X_{\infty}\right)=\mathbb{E}\left(\sum_{n=0}^{\infty} X_{n} \Delta H_{n}^{o}\right)
$$

and, from property (7) of $H^{o}$, one has

$$
\mathbb{E}\left(H_{\infty-}^{o} X_{\infty}\right)=\mathbb{E}\left(X_{\tau} \mathbb{1}_{\{\tau<\infty\}}\right) .
$$

It follows that

$$
\begin{aligned}
X_{0} & =\mathbb{E}\left(X_{\tau}\right)=\mathbb{E}\left(X_{\tau} \mathbb{1}_{\{\tau<\infty\}}\right)+\mathbb{E}\left(X_{\infty} \mathbb{1}_{\{\tau=\infty\}}\right)=\mathbb{E}\left(X_{\tau} \mathbb{1}_{\{\tau<\infty\}}\right)+\mathbb{E}\left(X_{\infty}\right)-\mathbb{E}\left(X_{\infty} \mathbb{1}_{\{\tau<\infty\}}\right) \\
& =\mathbb{E}\left(H_{\infty-}^{o} X_{\infty}\right)+X_{0}-\mathbb{E}\left(X_{\infty} \mathbb{1}_{\{\tau<\infty\}}\right),
\end{aligned}
$$

hence $\mathbb{E}\left(H_{\infty-}^{o} X_{\infty}\right)=\mathbb{E}\left(X_{\infty} \mathbb{1}_{\{\tau<\infty\}}\right)=\mathbb{E}\left(X_{\infty} \mathbb{P}\left(\tau<\infty \mid \mathcal{F}_{\infty}\right)\right)$ which implies $H_{\infty-}^{o}=\mathbb{P}\left(\tau<\infty \mid \mathcal{F}_{\infty}\right)$.

(ii) $\Rightarrow$ (iii) Obvious

(iii) $\Rightarrow$ (iv) By definition of $H^{o}$, and (8), we have that

$$
\widetilde{M}_{n}=H_{n}^{0}+Z_{n}=H_{n-1}^{0}+\widetilde{Z}_{n}, \quad \forall n \geq 1,
$$

therefore, by (iii), we deduce that $\widetilde{Z}_{n}=1-H_{n-1}^{0}$ which, since $H^{0}$ is $\mathbb{F}$-adapted, is $\mathcal{F}_{n-1^{-}}$ measurable for all $n \geq 1$, i.e. $\widetilde{Z}$ is $\mathbb{F}$-predictable.

(iv) $\Rightarrow$ (v) If $\widetilde{Z}$ is predictable, $\widetilde{M}$ is a predictable martingale, hence a constant (indeed, $\left.\mathbb{E}\left(\widetilde{M}_{n} \mid \mathcal{F}_{n-1}\right)=\widetilde{M}_{n}=\widetilde{M}_{n-1}\right)$ and $\Delta\langle X, \widetilde{M}\rangle_{n}=0$ for all $n \geq 1$. The result follows from Proposition 3.11 .

(v) $\Rightarrow$ (i) For any bounded $\mathbb{F}$-martingale $X$, the stooped process $X^{\tau}$ is a $\mathbb{G}$-martingale. Then, as a consequence of the optional stopping theorem applied in $\mathbb{G}$ at time $\tau$, we get $\mathbb{E}\left(X_{\tau}\right)=\mathbb{E}\left(X_{0}\right)$, hence, $\tau$ is an $\mathbb{F}$ pseudo-stopping time.

Obviously, pseudo-stopping times do not create arbitrages before $\tau$. In continuous time, the links between pseudo-stopping times and immersion property are presented in [3], and it is proved that $\tau$ is a pseudo-stopping time if and only if $\widetilde{Z}$ is a càglád decreasing process. 


\section{References}

[1] Acciaio B. and Fontana, C. and Kardaras C., Arbitrage of the First Kind and Filtration Enlargements In Semimartingale Financial Models, Preprint, arxiv.org/pdf/1401.7198.pdf, 2014.

[2] Aksamit, A., Random times, enlargement of filtration and arbitrages, Thèse de Doctorat, Univ. Evry, 2014.

[3] Aksamit, A., and Libo, L. Pseudo-stopping times and the Immersion property, Preprint, arxiv.org/abs/1409.0298 2015.

[4] Aksamit, A., Choulli, T. and Deng, J. and Jeanblanc, M., Non-Arbitrage up to Random Horizon for Semimartingale Models, Preprint, arXiv:1310.1142, 2014.

[5] Barlow, M.T. Study of filtration expanded to include an honest time Z. Wahr. Verw. Gebiete, 44, 307-323, 1978.

[6] Choulli, T., Daveloose, C. and Vanmaele, M. : Hedging mortality risk and optional martingale representation theorem for enlarged filtration. Preprint, arXiv:1510.05858, 2015.

[7] Choulli, T. and Deng, J. Non-arbitrage for Informational Discrete Time Market Models Preprint, arxiv.org/pdf/1407.1453. 2014.

[8] Nikeghbali, A. and Yor, M., A definition and some properties of pseudo-stopping times, The Annals of Probability, 33, 1804-1824, 2005.

[9] Romo, Romero R. Thèse de Doctorat, Univ. Evry. In preparation, 2016.

[10] Song, S, Dynamic one-default model, Arbitrage, Credit and Informational Risks, Proceedings of the Sino-French Research Program in Financial Mathematics Conference, World Scientific, 119-1,7 2014. Available on arxiv.org/abs/1309.7635. 